\title{
ON THE DIFFERENTIAL GEOMETRY OF HOMOGENEOUS VECTOR BUNDLES
}

\author{
BY \\ PHILLIP A. GRIFFITHS
}

This paper falls roughly into three parts. The first section (§§I-III) may be considered as an extension of the works of Nomizu and Wang [15; 19] to bundles over homogeneous complex manifolds. These results lay the groundwork for the succeeding paragraphs. The second section ( $\S$ IV-VI) gives differential-geometric derivations of various properties of homogeneous complex manifolds. The last section ( $\S \mathrm{VII}-\mathrm{IX})$ gives some applications of differential geometry to homogeneous vector bundles in the sense of [4] and to the study of sheaf cohomology.

To be more explicit, we let $X$ be a homogeneous complex manifold which may be written as the coset space of complex Lie groups $A, B(X=A / B)$ and also as the coset space of compact Lie groups $M, V(X=M / V)$ where $M$ is semi-simple. The first part treats the following question: If $P \rightarrow X$ is any analytic principal bundle to which the action of $A$ on $X$ lifts, we ask for a computable algebraic description of those connexions $\chi$ in $P$ which are $M$-invariant and compatible with the complex structures involved. (It will be seen that the adjective "computable", is for us crucial.) Moreover, given such a $\chi$, we seek its curvature form, and, in the case where $P$ is the principal tangent bundle, we ask for the complex torsion of $\chi$.

In the second part, we single out two types of connexions in such a $P$ for further study. The first are those connexions arising from an invariant Hermitian metric in $P$. By examining these connexions, we are able to give a unified differentialgeometric treatment of several aspects in the theory of homogeneous complex manifolds. The complex torsion plays an important role here.The other connexion, which we call the canonical complex connexion, is the complex analogue of the Nomizu canonical affine connexion; it is important for applications to sheaf theory.

In the third part, we first give a geometric realization of the "curvature class", a sheaf cohomology class defined by Atiyah in [1]. Next, using a metric geometry, we discuss a "vanishing theorem"; the chief application here is the non-Kähler case. In the last paragraph, we give a new type of application of differential geometry to sheaf cohomology; this application arises from a connextion between the holonomy algebra of a linear connexion and the coboundary maps in an exact cohomology sequence.

Received by the editors March 20, 1962 and, in revised form, May 20, 1962. 
Two remarks on the nature of the differential-geometric problems involved when $A / B$ is a complex manifold are pertinent. We shall eventually consider homogeneous complex manifolds which may be written as $M / V$ where $M, V$ are compact Lie groups and may also be written as $A / B$ where $A, B$ are complex Lie groups; say $X=M / V=A / B$. Then if $G$ is a complex Lie group and $G \rightarrow P \rightarrow X$ is an analytic principal fibre bundle to which the actions of $M$ and $A$ on $X$ lift, it is too much to require that a differential-geometric structure be $A$ invariant (e.g., $T\left(P_{n}(\mathbf{C})\right.$ ). Thus we are led to analyze those connexions in $P$ which are $M$-invariant and are "compatible" with $A$, i.e., respect the complex structure on $P$. Also one knows that on $T(A / B)$ we may define the complex torsion [2] whose vanishing is related to the Kähler condition on $X$. To our knowledge, this torsion has not been exhibited on a class of spaces, and by doing so here we recover the condition of Borel-Lichnerowicz that $A / B$ be Kähler.

We close with a few words on notation and conventions to be adopted. All manifolds, mappings, etc., will be $C^{\infty}$; if $X$ is a manifold, its tangent bundle is denoted by $T(X)$. A general vector bundle over $X$ will be written as $E \rightarrow \mathbf{E} \rightarrow X$ where $E$ is a vector space representing a typical fibre. If $E$ is a vector space, $E^{\prime}$ will be its dual and thus $E^{\prime} \rightarrow \mathbf{E}^{\prime} \rightarrow X$ will be the vector bundle dual to $E \rightarrow \mathbf{E} \rightarrow X$. In general, all manifolds and groups will be assumed to be connected. If $V$ is a vector space over a field $K$ and if $V^{\prime} \subset V$ is a subset, we denote by ${ }_{K}\left(V^{\prime}\right)$ the smallest linear subspace of $V$ containing $V^{\prime}$. We denote by $\operatorname{GL}(V)$ the group of automorphisms of $V$ and by $\operatorname{gl}(V)$ the algebra of endomorphisms of $V$. If again $V$ is a vector space over the reals $\mathbf{R}$ or the rationals $\mathbf{Q}$, we denote the complexification $V \otimes C_{\mathrm{R}}$ or $V \otimes_{\mathrm{Q}} C$ by $\tilde{V}$. If $A$ is a Lie group, we denote by $\mathfrak{a}^{\circ}$ the real Lie algebra of $A$ and by $\tilde{\mathfrak{a}}^{\circ}$ the algebra complexification of $\mathfrak{a}^{\circ}$. If $G$ is a complex Lie group, we denote by $g$ the complex Lie algebra of $G$. As usual, "exp" denotes the exponential mapping. We remark that although in general, a representation of the Lie algebra does not allow us to recover a representation of the group, we shall ignore this in certain cases (e.g., Theorem 1); for economy of notation, we shall write equations in the algebra and state results for groupsin all cases this will be permissible.

Whenever $X$ is a complex manifold, the complexified tangent bundle splits into type components: $\tilde{T}(X)=T(X) \otimes_{\mathbf{R}} \mathbf{C} \cong \mathscr{T}(X) \oplus \overline{\mathscr{T}(X)}$ where $\mathscr{T}(X)$ denotes the bundle of vectors of type $(1,0)$. In particular, for a complex Lie group $G$, we write $\tilde{\mathfrak{g}}^{\mathrm{c}}=\mathfrak{g}_{h} \oplus \mathfrak{g}_{\bar{h}}$. If again $X$ is a complex manifold, we denote by $J_{X}$ the almost-complex structure tensor.

For background material, we suggest the following references: [16] for the general differential-geometric background; [6] and [7] for the relationship between curvature and characteristic classes; and [9] or [18] for a discussion of those homogeneous complex manifolds with which we shall be mainly con- 
cerned. Finally we remark that this paper is intended to accompany another work [8] in which the results obtained here will be used frequently.

I. Invariant connexions in homogeneous vector bundles. Let $G$ be a Lie group and $G \rightarrow P \rightarrow X$ a principal fibration over a manifold $X$. We recall that a connexion $\chi$ in $P$ is given either by a right-invariant $C^{\infty}$ distribution of horizontal spaces, which we write $p \rightarrow H(p) \subset T_{p}(P)(p \in P)$, or by a $\mathrm{g}^{\circ}$-valued form $\omega$ on $P$ satisfying $\left(R_{g}\right)^{*} \omega=\left(\operatorname{Ad~} g^{-1} \omega\right)(g \in G)$. The connexion form $\omega$ appears as a bonafide form on $P$; however, $\omega$ comes from a nontensorial form $\theta$ on $X$ and we may symbolically write

$$
\omega=g^{-1} d g+\left(\operatorname{Ad} g^{-1}\right) \theta .
$$

Later on we shall study holomorphic fibrations $G \rightarrow P \rightarrow X$ where all manifolds and maps are complex analytic. If $J_{Y}$ represents the almost complex structure tensor of an appropriate object $Y$, we define a complex connexion $\chi$ in $P$ to be a real connexion $\chi$ such that:

$$
J_{P}(p) H(p)=H(p) J_{P}(p) \quad(p \in P) .
$$

It is easily checked that (i) is equivalent to:

$$
J_{G} \omega=\omega J_{P}
$$

Now let $G \rightarrow P \rightarrow X$ be a holomorphic fibration, $\chi$ a connexion in $P$ extended by linearity to $(T(P))^{\sim}=T(P) \otimes_{\mathrm{R}} \mathrm{C} \cong \mathscr{T}(P) \oplus \overline{\mathscr{T}(P)}$, and denote the extension of $H$ by $\mathscr{H}$.

Proposition 1.1. The following are necessary and sufficient that $\chi$ be $a$ complex connexion:

(i) $\mathscr{H}: \mathscr{T}(P) \rightarrow \mathscr{T}(P)$ and $\mathscr{H}(\bar{v})=\overline{\mathscr{H}(v)}$ for $v \in T(P)$;

(ii) $\mathscr{H}(p)=\mathscr{H}_{h}(p) \oplus \overline{\mathscr{H}_{h}(p)}$ is a splitting of the horizontal spaces into conjugate subspaces;

(iii) $\omega: \mathscr{T}(P) \rightarrow g_{h}$ and $\omega(\bar{v})=\overline{\omega(v)}$;

(iv) if $\theta$ is given by (1.1), $\theta=\theta_{1,0}+\theta_{0,1}$ where $\bar{\theta}_{0,1}=\theta_{1,0}$ and $\theta_{1,0}$ is a local form of type $(1,0)$ on $X$.

Proof. The proof is easy from the definitions together with the fact that, if $Y$ is a complex manifold, $\mathscr{T}_{y}(Y)$ is the set of vectors of the form $\left(\lambda v-i J_{Y}(y) \lambda v\right)$ for $v \in T_{y}(Y), \lambda \in \mathbf{C}$.

Throughout the rest of $\S \mathrm{I}$, we shall work in the real case and shall return to the complex situation in $\S I$ II. Let $X$ be a manifold on which a Lie group $A$ acts transitively; then we write $X=A / B$ where $B$ is the stability group of a point $x_{0}$ on $X$. A bundle $G \rightarrow P \stackrel{\pi}{\rightarrow} X$ such that $A$ acts on $P$ in such a manner that the following diagram commutes 




will be called a homogeneous bundle. We then have that $b\left(\pi^{-1}\left(x_{0}\right)\right) \subseteq \pi^{-1}\left(x_{0}\right)$ for all $b \in B$, and we may define a homomorphism $\phi: B \rightarrow G$ as follows: if $p_{0} \in \pi^{-1}\left(x_{0}\right)$ is fixed once and for all, then $b p_{0}=p_{0} g_{b}$ for some $g_{b} \in G$ and the mapping $\phi(b)=g_{b}$ is a homomorphism. We thus have

$$
\phi: B \rightarrow G \text { by } \phi(b)=g_{b} \text { and } \phi_{*}: \mathfrak{b}^{\circ} \rightarrow \mathfrak{g}^{\circ} .
$$

Definition 1.1. A connexion $\chi$ in $P$ is $A$-invariant if, for any $p \in P, a \in A$, $a_{*} H(p) \subseteq H(a p)$. This condition is easily equivalent to $a^{*} \omega=\omega$ where $\omega$ is the connexion form.

Thus the invariant connexions are characterized by

$$
a_{*} H=H a_{*} \Leftrightarrow a^{*} \omega=\omega .
$$

We define a mapping $\sigma: A \rightarrow P$ by

$$
\sigma(a)=a p_{0} .
$$

If $\omega$ is a connexion form on $P$, then $\sigma^{*} \omega$ is a 1 -form on $A$ with values in $g^{\circ}$ and we have

Proposition 1.2. Let $t \in \mathfrak{a}^{\circ}, b \in B^{\circ}$. Then, if $\omega$ is A-invariant,

(i) $\sigma^{*} \omega(\operatorname{Ad} b(t))=\operatorname{Ad} \phi(b)\left(\sigma^{*} \omega(t)\right)$;

(ii) $v \in \mathfrak{b}^{\circ} \Rightarrow \sigma^{*} \omega(v)=\phi_{*}(v) \in \mathfrak{g}^{\circ}$.

Proof. The proof is done by a calculation using the definitions; we refer to $[19, \S 5]$ for the proof.

The converse to Proposition 1.2 is also given in [19] and we have:

THEOREM W. The A-invariant linear connexions in the homogeneous bundle $G \rightarrow P \rightarrow A / B$ are given by the linear mappings $\chi: \mathfrak{a}^{\circ} \rightarrow \mathfrak{g}^{\circ}$ satisfying the conditions:

(i) $\chi \circ \mathrm{Ad}=(\operatorname{Ad} \circ \phi) \circ \chi$,

(ii) $\chi(v)=\phi_{*}(v)$ for $v \in \mathfrak{b}^{\circ}$.

Here $\phi$ is defined by (1.2); the relation between the connexion form $\omega$ and the mapping $\chi$ is $\sigma^{*} \omega \mid \mathfrak{a}^{\circ}=\chi$ where $\sigma$ is given by (1.4).

From the Cartan structure equation for the curvature form and the fact that $\sigma^{*} \omega$ is left invariant on $A$, it follows that the curvature $\Xi$, considered as an element of $\operatorname{Hom}\left(\mathfrak{a}^{\circ} \otimes \mathfrak{a}^{\circ}, \mathfrak{g}^{\circ}\right)$, is given by

$$
\Xi\left(t, t^{\prime}\right)=\frac{1}{2}\left\{\left[\chi(t), \chi\left(t^{\prime}\right)\right]-\chi\left[t, t^{\prime}\right]\right\} \quad\left(\text { for } t, t^{\prime} \in \mathfrak{a}^{\circ}\right) .
$$


Finally, since we shall be working primarily with Lie algebras instead of groups, we shall state the differential form of (1.5).

TheOREM 1. The A-invariant linear connexions in $G \rightarrow P \rightarrow A / B$ are given infinitesimally by the linear mappings $\chi: \mathfrak{a}^{\circ} \rightarrow \mathfrak{g}^{\circ}$ satisfying thc conditions:

$$
\begin{aligned}
\text { (i) } \chi[b, a] & =[\chi(b), \chi(a)] & & a \in \mathfrak{a}^{\circ}, \quad b \in \mathfrak{b}^{\circ}, \\
\text { (ii) } \chi(b) & =\phi_{*}(b) & b \in \mathfrak{b}^{\circ} . &
\end{aligned}
$$

The curvature form $\Xi \in \operatorname{Hom}\left(\mathfrak{a}^{\circ} \otimes \mathfrak{a}^{\circ}, \mathfrak{g}^{\circ}\right)$ is given by

$$
\Xi\left(a, a^{\prime}\right)=\frac{1}{2}\left\{\left[\chi(a), \chi\left(a^{\prime}\right)\right]-\chi\left[a, a^{\prime}\right]\right\} \quad a, a^{\prime} \in \mathfrak{a}^{\circ} .
$$

II. Invariant complex connexions in homogeneous analytic vector bundles. We now wish to examine the structures in analytic fibre bundles $G \rightarrow P \rightarrow A / B$ where $A / B$ is a complex homogeneous manifold. It will be assumed that $A / B$ is a $C$-space as defined in [18]; this means that $A / B$ is a compact homogeneous complex manifold with a finite fundamental group. For a while the essential property of a $C$-space $X$ which we shall use is the following: a $C$-space $X$ may be represented as the coset space of compact Lie groups $A, B ; X=A / B$ (compact description) or as the coset space of complex Lie groups $E, F ; X=E / F$ (complex description). Furthermore, we may and shall assume that all groups are connected, that $A, E$ are semi-simple, and that $E$ is the complexification of $A$. The theorems of the next two sections are true for homogeneous complex manifolds satisfying the requirements of the preceding sentence, although these results shall only be stated for $C$-spaces.

Let $X=A / B=E / F$ be a $C$-space where $A / B$ is the compact description and $E / F$ the complex form. Suppose that $G \rightarrow P \stackrel{\pi}{\rightarrow} X$ is a holomorphic principal fibration with the complex connected Lie group $G$ as structure group. As discussed above, we desire those connexions which are compatible with $E$ and $A$-invariant. Some delicacy is required here, for not just any solution will do. In fact we desire a formulation involving only the complex Lie algebras of $E$ and $G$ and the homomorphism $\phi_{*}$ defined in $§$ I. As will appear below, an answer in terms of the real Lie algebras is not difficult to obtain; however, such a solution is unsatisfactory in practice. (Witness how unwieldy the real Lie algebra $\operatorname{SL}(n, \mathbf{C})$ is.) With these remarks in mind, we begin.

In the analytic fibration $G \rightarrow P \stackrel{\pi}{\rightarrow} X$, we assume that the action of $E$ on $X$ lifts holomorphically to $P$, i.e., the diagram

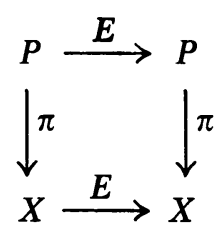


is commutative and analytic. Define the pair $\left(\sigma^{\prime}, \phi^{\prime}\right)$ for $(E, F)$ in the same manner as $(\sigma, \phi)$ for $(A, B) ;\left(\sigma^{\prime}, \phi^{\prime}\right)$ extends $(\sigma, \phi)$. The mapping $\sigma^{\prime}: E \rightarrow P$ is analytic, so that $J_{P} \sigma_{*}=\sigma_{*}^{\prime} J_{E}$. The equation for complex compatibility of a connexion $\omega$ on $P$ is $J_{G}{ }^{\circ} \omega=\omega \circ J_{P}$. Consider the mappings.

$$
\begin{aligned}
& \sigma^{*} \omega: \mathfrak{a}^{\circ} \rightarrow \mathfrak{g}^{\circ}=\chi: \mathfrak{a}^{\circ} \rightarrow \mathfrak{g}^{\circ}, \\
& \left(\sigma^{*} \omega: \varepsilon^{\circ} \rightarrow \mathfrak{g}^{\circ}=\chi^{\prime}: \varepsilon^{\circ} \rightarrow \mathfrak{g}^{\circ}\right.
\end{aligned}
$$

( $\varepsilon^{\circ}$ is the real Lie algebra of $E$ ). Take $\varepsilon \in \varepsilon^{\circ} ;$ then $\left(\sigma^{\prime}\right)_{*}(\varepsilon) \in T_{p_{0}}(P)$ and since $\sigma^{\prime}$ is analytic, $J_{P} \sigma_{*}^{\prime}(\varepsilon)=\sigma_{*}^{\prime} J_{E}(\varepsilon)$; thus

$$
\begin{aligned}
\chi^{\prime}\left(J_{E} \varepsilon\right) & =\left(\sigma^{\prime}\right)_{*} \omega\left(J_{E} \varepsilon\right)=\omega\left(\sigma_{*}^{\prime} J_{E} \varepsilon\right) \\
& =\omega\left(J_{P} \sigma_{*}^{\prime} \varepsilon\right)=J_{G} \omega\left(\sigma_{*}^{\prime} \varepsilon\right) \\
& =J_{G}\left(\sigma^{\prime}\right)^{*} \omega(\varepsilon)=J_{G} \chi^{\prime}(\varepsilon)
\end{aligned}
$$

Proposition 2.1. The equation of complex compatibility for the connexion $\chi$ is

$$
\chi^{\prime} J_{E}=J_{G} \chi^{\prime}
$$

Recall that $\sim$ denotes the complexification of a real Lie algebra, i.e., $\mathfrak{a}^{\circ} \otimes_{\mathrm{R}} \mathbf{C}=\tilde{\mathfrak{a}}^{\circ}$. Extend $\chi^{\prime}$ to $\tilde{\boldsymbol{\varepsilon}}^{\circ} \cong \varepsilon_{h} \oplus \varepsilon_{\bar{h}}$ by complex linearity. Then (cf. Proposition 1.1)

$$
\begin{aligned}
& \chi^{\prime}: \varepsilon_{h} \rightarrow \mathfrak{g}_{h}, \\
& \chi^{\prime}: \varepsilon_{\bar{h}} \rightarrow \mathfrak{g}_{\bar{h}} \quad \text { and } \quad \chi^{\prime}(\bar{\varepsilon})=\overline{\chi^{\prime}(\bar{\varepsilon}) .}
\end{aligned}
$$

There are two things which must be attended to:

(i) invariance under the compact group $A$;

(ii) compatibility with the complex group $E$.

The general situation is this: Let $X$ be a manifold and $G \rightarrow P \stackrel{\pi}{\rightarrow} X$ a principal fibration and assume the existence of

(i) a fibre-transitive group $E$ on $P$ with isotropy group $F$,

(ii) a fibre-transitive group $A \subseteq E$ on $P$ with isotropy group $B \subseteq F$. Then $X=A / B=E / F$ and we may define $(\sigma, \phi)$ and $\left(\sigma^{\prime}, \phi^{\prime}\right)$. Let $\omega$ be an $A$-invariant connexion on $P$; then $\omega: T(P) \rightarrow \mathrm{g}^{\circ}$ and $\left(\sigma^{\prime}\right)^{*} \omega: T(E) \rightarrow \mathrm{g}^{\circ}$ gives (by restriction to $\left.\varepsilon^{\circ}\right) \chi^{\prime}: \varepsilon^{\circ} \rightarrow g^{\circ}$. Furthermore, $\sigma^{*} \omega: T(A) \rightarrow g^{\circ}$ restricts to $\chi: a^{\circ} \rightarrow g^{\circ}$ and $\chi^{\prime}$ extends $\chi$ from $\mathfrak{a}^{\circ}$ to $\varepsilon^{\circ}$. Invariance under $A$ means that

$$
\begin{aligned}
\chi^{\prime}[a, b] & =\chi[a, b]=[\chi(a), \chi(b)] \\
& =\left[\chi^{\prime}(a), \chi^{\prime}(b)\right] \quad a \in \mathfrak{a}^{\circ}, b \in \mathfrak{b}^{\circ} .
\end{aligned}
$$

Since $\omega$ is a connexion, we also may say that $\chi^{\prime}(f)=\phi_{*}^{\prime}(f)$ for $f \in \mathfrak{f}^{\circ}$; in particular, $\chi^{\prime}(b)=\chi(b)=\phi_{*}^{\prime}(b)=\phi_{*}(b)$ for $b \in \mathfrak{b}^{\circ} \subseteq \mathfrak{f}^{\circ}$. 
Returning now to the case of $C$-spaces where everything is complex (this being a special case of the above), we wish $\chi^{\prime}$ to be a complex connexion, i.e.,

$$
\begin{aligned}
\chi^{\prime}: \varepsilon^{\circ} & \rightarrow \mathfrak{g}^{\circ}, \\
\chi^{\prime} \mid \mathfrak{a}^{\circ}=\chi: \mathfrak{a}^{\circ} & \rightarrow \mathfrak{g}^{\circ}
\end{aligned}
$$

and

$$
\chi^{\prime} J_{E}=J_{G} \chi^{\prime}
$$

Since $A$ is by assumption a maximal compact subgroup of $E, \varepsilon^{\circ}=\mathfrak{a}^{\circ} \oplus J_{E} \mathfrak{a}^{\circ}$ and hence $\chi^{\prime}$ is uniquely determined by $\chi$. But every $\chi$ does not give a $\chi^{\prime}$ because of the condition $\chi^{\prime} \mid \mathfrak{f}^{\circ}=\phi_{*}^{\prime}$. Indeed, if every $\chi$ uniquely extended to such a complex $\chi^{\prime}$, this would mean that every real connexion on $A$ was complex. This, however, is false; simple examples appear below. In an effort to trap both complex compatibility and $A$-invariance in a single computable expression, we proceed as follows.

Definition. Define a linear mapping $\rho: \tilde{\mathfrak{a}}^{\circ} \rightarrow \varepsilon_{h}$ by

$$
\rho(a)=\frac{1}{2}\left(a+i J_{E} a\right) \quad \text { for } a \in \tilde{\mathfrak{a}}^{\circ} .
$$

That $\rho$ takes $\tilde{\mathfrak{a}}^{\circ}$ into $\varepsilon_{h}$ follows from the discussion in $\S$. Both $\mathfrak{a}^{\circ}$ and $\boldsymbol{\varepsilon}_{\boldsymbol{h}}$ are complex Lie algebras and we shall prove below that $\rho$ is an algebra isomorphism. Before doing this, an example might be instructive.

Let $A=U(n)$ and $E=G=\operatorname{GL}(n, \mathbf{C})$. Then $\mathfrak{a}^{\circ}=$ the (real) algebra of $n \times n$ skew-Hermitian matrices; any $\alpha \in \mathfrak{a}^{\circ}$ may be written as $\alpha=\beta+i \gamma$, where $\beta$ is real skew-symmetric and $\gamma$ is real symmetric. On the other hand, $\operatorname{GL}(n, \mathbf{C})$ $\subset \mathrm{GL}(2 n, \mathbf{R})$ and $\mathrm{g}^{\circ}$ may be represented as the sub-algebra of $\mathrm{gl}(2 n, \mathbf{R})$ of matrices of the form

$$
\left(\begin{array}{rr}
\eta & \tau \\
-\tau & \eta
\end{array}\right)
$$

where $\eta, \tau \in \operatorname{gl}(n, \mathbf{R})$. Furthermore, we may represent $J_{\mathbf{G L}(n, \mathbf{C})}=J$ as the matrix

$$
\begin{gathered}
\left(\begin{array}{rr}
0 & -1 \\
1 & 0
\end{array}\right) ; \\
J^{2}=\left(\begin{array}{rr}
0 & -1 \\
1 & 0
\end{array}\right)\left(\begin{array}{rr}
0 & -1 \\
1 & 0
\end{array}\right)=\left(\begin{array}{rr}
-1 & 0 \\
0 & -1
\end{array}\right)
\end{gathered}
$$

and

$$
J\left(\begin{array}{rr}
\eta & \tau \\
-\tau & \eta
\end{array}\right)=\left(\begin{array}{rr}
0 & -1 \\
1 & 0
\end{array}\right) \quad\left(\begin{array}{rr}
\eta & \tau \\
-\tau & \eta
\end{array}\right)=\left(\begin{array}{rr}
\tau & -\eta \\
\eta & \tau
\end{array}\right)
$$

We first check that $\mathfrak{g}^{\circ}=\mathfrak{a}^{\circ} \oplus J \mathfrak{a}^{\circ}$; remark, however, that $\alpha=\beta+i \gamma \in \mathfrak{a}^{\circ}=\mathfrak{u}(n)$ must be written in the form 


$$
\alpha=\left(\begin{array}{rr}
\beta & \gamma \\
-\gamma & \beta
\end{array}\right)
$$

when $U(n)$ is considered as lying in $\operatorname{GL}(n, \mathbf{C})$.

(i) $\mathfrak{u}(n) \cap J(\mathfrak{u}(n))=0$ : let $\alpha \in \mathfrak{u}(n) \cap J(\mathfrak{u}(n))$; then

$$
\alpha=\left(\begin{array}{rr}
\beta & \gamma \\
-\gamma & \beta
\end{array}\right)=J\left(\begin{array}{rr}
\beta^{\prime} & \gamma^{\prime} \\
-\gamma^{\prime} & \beta^{\prime}
\end{array}\right)=\left(\begin{array}{rr}
\gamma^{\prime} & -\beta^{\prime} \\
\beta^{\prime} & \gamma^{\prime}
\end{array}\right)
$$

or $\beta=\gamma^{\prime}, \gamma=-\beta^{\prime}$ where $\beta, \beta^{\prime}$ are real skew-symmetric; $\gamma, \gamma^{\prime}$ are real symmetric. Thus $\mathfrak{u}(n) \cap J(\mathfrak{u}(n))=0$.

(ii) $\mathfrak{g}^{\circ}=\mathfrak{u}(n) \oplus J(\mathfrak{u}(n))$. Now $\mathfrak{u}(n) \oplus J(\mathfrak{u}(n)) \subseteq \mathfrak{g}^{\circ}$ and from dimensional considerations, we are done. However, it is of interest to give an explicit proof. Given

we wish to write

$$
\left(\begin{array}{ll}
\eta & \tau \\
\tau & \eta
\end{array}\right)
$$

$$
\left(\begin{array}{rr}
\eta & \tau \\
-\tau & \eta
\end{array}\right)=\left(\begin{array}{rr}
\beta & \gamma \\
-\gamma & \beta
\end{array}\right)+\left(\begin{array}{rr}
\gamma^{\prime} & -\beta^{\prime} \\
\beta^{\prime} & \gamma^{\prime}
\end{array}\right)
$$

where $\beta, \beta^{\prime}$ are skew-symmetric, $\gamma, \gamma^{\prime}$ are symmetric. The statement that $\eta=\beta+\gamma^{\prime}, \tau=\gamma-\beta^{\prime}$ is just the well-known statement that a real matrix may be (uniquely) written as the sum of a symmetric matrix and a skew-symmetric matrix; $A=\frac{1}{2}\left(A+{ }^{t} A\right)+\frac{1}{2}\left(A-{ }^{t} A\right)\left({ }^{1}\right)$.

Now $(\mathfrak{u}(n))^{\sim}=\mathfrak{u}(n) \otimes_{\mathrm{R}} \mathbf{C}=$ the set of matrices

$$
\left\{\left(\begin{array}{rr}
\beta & \gamma \\
-\gamma & \beta
\end{array}\right)\right\}
$$

where $\beta$ is complex skew-symmetric and $\gamma$ is complex symmetric. Furthe:-..ore,

$$
\tilde{\mathfrak{g}}^{\circ}=\left\{\left(\begin{array}{rr}
\eta & \tau \\
-\tau & \eta
\end{array}\right)\right\}
$$

$\eta, \tau \in \operatorname{gl}(n, \mathbf{C})$. We may isolate $\mathfrak{g}_{h}$ and $\mathfrak{g}_{\bar{h}}$ :

$$
\Pi_{1,0}\left(\begin{array}{ll}
\eta & \tau \\
\tau & \eta
\end{array}\right)=\frac{1}{2}\left\{\left(\begin{array}{rr}
\eta & \tau \\
-\tau & \eta
\end{array}\right)+i\left(\begin{array}{rr}
\tau & -\eta \\
\eta & \tau
\end{array}\right)\right\}=\left(\begin{array}{rr}
\eta^{\prime} & -i \eta^{\prime} \\
i \eta^{\prime} & \eta^{\prime}
\end{array}\right),
$$

$\eta^{\prime}=\frac{1}{2}(\eta+i \tau)$ and

$$
\mathfrak{g}_{h}=\left\{\left(\begin{array}{cc}
\eta & -i \eta \\
i \eta & \eta
\end{array}\right)\right\}
$$

for $\eta \in \operatorname{gl}(n, \mathbf{C})$. Similarly,

(1) The statement precisely corresponds (in the sense of Lie algebras) to the statement that $a \in \mathrm{gl}(n, \mathrm{C})$ may be uniquely written as the sum of an Hermitian plus a skew-Hermitian matrix. 


$$
\mathfrak{g}_{\bar{h}}=\left\{\left(\begin{array}{rr}
\eta & i \eta \\
-i \eta & \eta
\end{array}\right)\right\}
$$

for $\eta \in \operatorname{gl}(n, \mathbf{C})$ and $\left[\mathfrak{g}_{h}, \mathfrak{g}_{h}\right]=0$. Furthermore, $(\mathfrak{u}(n)) \sim g \mathrm{gl}(n, \mathbf{C}) \cong \mathfrak{g}_{h}$; the first isomorphism is the footnote above; the second is given now. Define $\rho:(\mathfrak{u}(n))^{\sim} \rightarrow \mathrm{g}_{h}$ by

$$
\begin{aligned}
\rho(\alpha) & =\rho(\beta+i \gamma)=\rho\left(\begin{array}{rr}
\beta & \gamma \\
-\gamma & \beta
\end{array}\right) \\
& =\frac{1}{2}\left\{\left(\begin{array}{rr}
\beta & \gamma \\
-\gamma & \beta
\end{array}\right)+i\left(\begin{array}{cc}
\gamma & \beta \\
-\beta & \gamma
\end{array}\right)\right\}=\frac{1}{2}\left(\begin{array}{rr}
\alpha & -i \alpha \\
i \alpha & \alpha
\end{array}\right)
\end{aligned}
$$

where $\beta$ and $\gamma$ are the symmetric and skew-symmetric parts of $\alpha \in \operatorname{gl}(n, \mathbf{C})$. We see that $\rho$ is simply given by $\rho(\alpha)=\frac{1}{2}(\alpha+i J \alpha)$. One checks that

$$
\begin{aligned}
& \alpha=\beta+i \gamma \rightarrow\left(\begin{array}{rr}
\beta & \gamma \\
-\gamma & \beta
\end{array}\right) \text { is a Lie algebra isomorphism, } \\
& \alpha \rightarrow-\frac{1}{2}\left(\begin{array}{rr}
\alpha & -i \alpha \\
i \alpha & \alpha
\end{array}\right) \text { is a Lie algebra isomorphism. }
\end{aligned}
$$

These are both special cases of

LEMMA 2.1. Let E be a complex semi-simple Lie group and $A$ a maximal compact subgroup. Then $\tilde{\mathfrak{a}}^{\circ} \cong \varepsilon_{h}$ and the isomorphism given by $\rho(a)=\frac{1}{2}\left(a+i J_{E} a\right)$ for $a \in \tilde{\mathfrak{a}}^{\circ}$.

Proof. $\rho\left[a, a^{\prime}\right]=\frac{1}{2}\left\{\left[a, a^{\prime}\right]+i J_{E}\left[a, a^{\prime}\right]\right\}$ and $\left[\rho(a), \rho\left(a^{\prime}\right)\right]=\frac{1}{4}\left\{\left[a, a^{\prime}\right]+i\left[J_{E} a, a^{\prime}\right]\right.$ $\left.+i\left[a, J_{E} a^{\prime}\right]-\left[J_{E} a, J_{E} a^{\prime}\right]\right\}$. Since $J_{E}$ is an integrable almost complex structure, $\left[a, a^{\prime}\right]+J_{E}\left[J_{E} a, a^{\prime}\right]+J_{E}\left[a, J_{E} a^{\prime}\right]-\left[J_{E} a, J_{E} a^{\prime}\right]=0$; hence $i\left[J_{E} a, a^{\prime}\right]+i\left[a, J_{E} a^{\prime}\right]$ $=i J_{E}\left[a, a^{\prime}\right]-i J_{E}\left[J_{E} a, J_{E} a^{\prime}\right]$. Thus

$$
\begin{aligned}
{\left[\rho(a), \rho\left(a^{\prime}\right)\right] } & =\frac{1}{2}\left\{\left[a, a^{\prime}\right]-\left[J_{E} a, J_{E} a^{\prime}\right]+i J_{E}\left[a, a^{\prime}\right]-i J_{E}\left[J_{E} a, J_{E} a^{\prime}\right]\right\} \\
& =\frac{1}{2}\left\{\rho\left[a, a^{\prime}\right]-\rho\left[J_{E} a, J_{E} a^{\prime}\right]\right\} .
\end{aligned}
$$

On the other hand, $\left[\varepsilon_{h}, \varepsilon_{h}\right]\left({ }^{2}\right)=0$, which implies that

$$
0=\left[a+i J_{E} a, a^{\prime}-i J_{E} a^{\prime}\right]=\left[a, a^{\prime}\right]+i\left[J_{E} a, a^{\prime}\right]-i\left[a, J_{E} a^{\prime}\right]+\left[J_{E} a, J_{E} a^{\prime}\right] .
$$

Now taking $a, a^{\prime}$ to be real (i.e., $a, a^{\prime} \in \mathfrak{a}^{\circ}$ ), we see that $\left[a, a^{\prime}\right]=-\left[J_{E} a, J_{E} a^{\prime}\right]$; this equation remains true on $\tilde{\mathfrak{a}}^{\circ}$ by complex linearity. We conclude that

$$
\begin{aligned}
{\left[\rho(a), \rho\left(a^{\prime}\right)\right] } & =\frac{1}{2}\left\{\rho\left[a, a^{\prime}\right]+\rho\left[a, a^{\prime}\right]\right\} \\
& =\rho\left[a, a^{\prime}\right] \text { for } a, a^{\prime} \in \mathfrak{a}^{\circ} .
\end{aligned}
$$

(2) This is clear, since if $z_{1}, \cdots, z_{n}$ is a complex canonical coordinate system for $E$ and $X \in \varepsilon_{h}, Y \in \varepsilon_{\vec{h}}$ then there exists local holomorphic functions $f_{x}^{i}$ and $f_{y}^{j}$ such that $\left.\left.X=\sum_{i} f_{x}^{i}(0) \partial / \partial z_{i}\right]_{z=0}, Y=\sum_{j} f_{y}^{j}(0) \partial / \partial z_{j}\right]_{z=0}$. 
Thus $\rho$ is a homomorphism; restricted to $\mathfrak{a}^{\circ}$, it clearly is injective and thus has no kernel on $\mathfrak{a}^{\circ}$. Finally, since $\operatorname{dim} \tilde{\boldsymbol{\varepsilon}}^{\circ}=2 \operatorname{dim} \tilde{\mathfrak{a}}^{\circ}$, for reasons of dimensionality $\rho$ is surjective. Q.E.D.

We need one further lemma.

LEMMA 2.2. Let $G \rightarrow P \rightarrow X$ be a complex principal fibration over a complex manifold $X$. Let $\mathfrak{f}^{\circ} \subseteq \mathfrak{g}^{\circ}$ be a sub-algebra such that $J_{G}\left(\mathfrak{f}^{\circ}\right) \cap \mathfrak{f}^{\circ}=0$. If $\omega$ is a complex connexion on $P$ with the property that $\omega(x)$ lies in $\mathfrak{f}^{\circ}$ for all $x \in T(P)$, then the curvature $\Omega$ of $\omega$ satisfies $\Omega(x, y)=\Omega\left(J_{P} x, J_{P} y\right)$ for all $x, y \in T(P)$.

Proof. Using the Cartan structure equation, it will suffice to know: for $x, y \in H(p), \omega([x, y])=\omega\left(\left[J_{P}(x), J_{P}(y)\right]\right)$. But since $\omega$ is a complex connexion and $J_{P}$ is integrable,

$$
-\omega([x, y])+\omega\left(\left[J_{P} x, J_{P} y\right]\right)=J_{P}\left\{\omega\left(\left[J_{P} x, y\right]\right)+\omega\left(\left[x, J_{P} y\right]\right)\right\}
$$

and we are done.

This lemma holds, in particular, for metric connexions (example above Lemma 2.1). For all complex connexions which we shall consider, it either follows from Lemma 2.2 or may be checked directly that $\Omega(x, y)=\Omega\left(J_{P} x, J_{P} y\right)$; in any event this condition on $\Omega$ (and hence on $\omega$ ) will be assumed henceforth.

REMARK. Lemma 2.2 is equivalent to saying that $\Omega$ is of type (1.1). Indeed, assume that $\Omega(x, y)=\Omega\left(J_{P} x, J_{P} y\right)$ for tangent vectors $x, y$. Then

$$
\Omega\left(x+i J_{P} x, y+i J_{P} y\right)=\Omega(x, y)-\Omega(x, y)+i\left\{\Omega\left(x, J_{P} y\right)+\Omega\left(J_{P} x, y\right)\right\}=0
$$

since $\Omega\left(x, J_{P} y\right)=-\Omega\left(J_{P} x, y\right)$. Conversely, if $\Omega\left(x+i J_{P} x, y+i J_{P} y\right)=0$, then

$$
\Omega(x, y)-\Omega\left(J_{P} x, J_{P} y\right)+i\left\{\Omega\left(x, J_{P} y\right)+\Omega\left(J_{P} x, y\right)\right\}=0 ;
$$

taking $x$ and $y$ to be real, we see that $\Omega(x, y)=\Omega\left(J_{P} x, J_{P} y\right)$.

We return now to the case of a $C$-space $X=A / B=E / F$. In computing the curvature form $\Xi$ on $\mathfrak{a}^{\circ}$ in $\S \mathrm{I}$, we used the fact that $\sigma^{*} \omega$ was left-invariant on $A$; this in turn was equivalent to $A$-invariance of $\omega$ on $P$. However, since $\mathfrak{a}^{\circ} \oplus J_{E} \mathfrak{a}^{\circ}=\varepsilon^{\circ}$ and $\Xi\left(J_{E} a, J_{E} a^{\prime}\right)=\Xi\left(a, a^{\prime}\right), \Xi\left(a, J_{E} a^{\prime}\right)=-\Xi\left(J_{E} a, a^{\prime}\right)$ we shall be able to compute $\Xi$ on $\varepsilon^{\circ}$, at the appropriate time.

Now our original goal was to get everything on $\tilde{\mathfrak{a}}^{\circ}$ (not on $\tilde{\boldsymbol{\varepsilon}}^{\circ}$ ); furthermore, the obvious way of extending $\chi$ on $\mathfrak{a}^{\circ}$ to $\tilde{\mathfrak{a}}^{\circ}$ by complex linearity is insufficient. This was the precise reason for introducing $\rho$.

Definition. Define $\tilde{\chi}: \tilde{\mathfrak{a}}^{\circ} \rightarrow \mathfrak{g}_{h}$ by $\tilde{\chi}(a)=\chi^{\prime}(\rho(a))$ for $a \in \tilde{\mathfrak{a}}^{\circ}$.

Proposition 2.2. $\tilde{\chi}$ is a well-defined complex linear mapping.

Proof. We must compute $\tilde{\chi}(i a)$ two ways and show consistency. On the one hand, for $e \in \varepsilon_{h}, \tilde{\chi}\left(i \rho^{-1}(e)\right)=i \tilde{\chi}\left(\rho^{-1}(e)\right)=i \chi^{\prime}(e)=J_{G} \chi^{\prime}(e)$; also $\tilde{\chi}\left(i \rho^{-1}(e)\right)$ $=\tilde{\chi}\left(\rho^{-1}(i e)\right)=\tilde{\chi}\left(\rho^{-1} J_{E}(e)\right)=\chi^{\prime}\left(J_{E} e\right)=J_{G} \chi^{\prime}(e)$ which was to be shown. 
The following is the crucial proposition.

Proposition 2.3. For $a \in \tilde{\mathfrak{a}}^{\circ}, b \in \tilde{\mathfrak{b}}^{\circ}$,

$$
\tilde{\chi}[a, b]=[\tilde{\chi}(a), \tilde{\chi}(b)] .
$$

Proof.

$$
\begin{aligned}
\tilde{\chi}[a, b]= & \chi^{\prime}(\rho[a, b]) \\
= & \chi^{\prime}([\rho(a), \rho(b)]) \quad(\text { by Lemma 2.4) } \\
= & \chi^{\prime}[a, b]+i \chi^{\prime}\left[J_{E} a, b\right] \\
& +i \chi\left[a, J_{E} b\right]+\chi^{\prime}\left[J_{E} i a, J_{E} i b\right] . \\
{[\tilde{\chi}(a), \tilde{\chi}(b)]=} & {\left[\chi^{\prime}(\rho(a)), \chi^{\prime}(\rho(b))\right] } \\
= & {\left[\chi^{\prime}(a), \chi^{\prime}(b)\right]+i\left[J_{G} \chi^{\prime}(a), \chi^{\prime}(b)\right] } \\
& +i\left[\chi^{\prime}(a), J_{G} \chi^{\prime}(b)\right]+\left[J_{G} \chi^{\prime}(i a), J_{G} \chi^{\prime}(i b)\right] .
\end{aligned}
$$

Now $\left[\varepsilon_{h}, \varepsilon_{\bar{h}}\right]=0$ implies that $\left[a, a^{\prime}\right]+i\left[J_{E} a, a^{\prime}\right]-i\left[a, J_{E} a^{\prime}\right]-\left[J_{E} i a, J_{E} i a^{\prime}\right]$ $=0$, and since $\rho$ is a homomorphism,

$$
\frac{1}{4}\left\{\left[a, a^{\prime}\right]+i\left[J_{E} a, a^{\prime}\right]+i\left[a, J_{E} a^{\prime}\right]+\left[J_{E} i a, J_{E} i a^{\prime}\right]\right\}=\frac{1}{2}\left(\left[a, a^{\prime}\right]+J_{E} i\left[a, a^{\prime}\right]\right) ;
$$

thus

$$
\left[a, a^{\prime}\right]+\left[J_{E} i a, a^{\prime}\right]=\left[a, a^{\prime}\right]+J_{E} i\left[a, a^{\prime}\right]
$$

and

$$
\left[a, J_{E} i a^{\prime}\right]+\left[J_{E} i a, J_{E} i a^{\prime}\right]=\left[a, a^{\prime}\right]+J_{E} i\left[a, a^{\prime}\right] .
$$

Using these equations in (2.6) and (2.7), we obtain the result.

Proposition 2.4. $\tilde{\chi} \mid \rho^{-1}\left(\mathfrak{f}_{h}\right)=\phi_{*}^{\prime} \circ \rho$.

Proof. Let $f \in \mathfrak{f}_{h} ; f=a+i J_{E} a$ for some $a \in \tilde{\mathfrak{a}}^{\circ}$. Then

$$
\tilde{\chi}(a)=\tilde{\chi}\left(\rho^{-1}(f)\right)=\chi^{\prime}\left(a+i J_{E} a\right)=\phi_{*}^{\prime}\left(a+i J_{E} a\right)=\phi_{*}^{\prime} \circ \rho(a) \text { Q.E.D. }
$$

To complete the discussion in this section, we compute the curvature of our complex connexion $\chi^{\prime}$. We have that

$$
\begin{aligned}
\Xi\left(a+i J_{E} a, a^{\prime}-i J_{E} a^{\prime}\right)= & \Xi\left(a, a^{\prime}\right)+i \Xi\left(J_{E} a, a^{\prime}\right) \\
& -i \Xi\left(a, J_{E} a^{\prime}\right)+\Xi\left(J_{E} a, J_{E} a^{\prime}\right) \quad\left(\text { for } a, a^{\prime} \in \mathfrak{a}^{\circ}\right) \\
= & 2 \Xi\left(a, a^{\prime}\right)+2 i \Xi\left(J_{E} a, a^{\prime}\right) \\
= & 2 \Xi\left(a, a^{\prime}\right)+2 i J_{G} \Xi\left(a, a^{\prime}\right) .
\end{aligned}
$$


On the other hand,

$$
\begin{gathered}
\begin{aligned}
& \frac{1}{2}\left\{\tilde{\chi}\left[a, a^{\prime}\right]-\left[\tilde{\chi}(a), \tilde{\chi}\left(a^{\prime}\right)\right]\right\}= \frac{1}{4}\left\{\chi^{\prime}\left[a, a^{\prime}\right]+i J_{G} \chi^{\prime}\left[a, a^{\prime}\right]\right. \\
&-\left[\chi^{\prime}(a), \chi^{\prime}\left(a^{\prime}\right)\right]-i\left[J_{G} \chi^{\prime}(a), \chi^{\prime}\left(a^{\prime}\right)\right] \\
&\left.-i\left[\chi^{\prime}(a), J_{G} \chi^{\prime}\left(a^{\prime}\right)\right]-\left[i J_{G} \chi^{\prime}(a), i J_{G} \chi^{\prime}\left(a^{\prime}\right)\right]\right\} \\
&=\frac{1}{4}\left\{\chi\left[a, a^{\prime}\right]+i J_{G} \chi\left[a, a^{\prime}\right]\right.\left.-\left[\chi(a), \chi\left(a^{\prime}\right)\right]-i J_{G}\left[\chi(a), \chi\left(a^{\prime}\right)\right]\right\} \\
&= \frac{1}{2}\left\{\Xi\left(a, a^{\prime}\right)+i J_{G} \Xi\left(a, a^{\prime}\right)\right\} . \\
&(2.8) \quad=2\left\{\tilde{\chi}\left[a, a^{\prime}\right]-\left[\tilde{\chi}(a), \tilde{\chi}\left(a^{\prime}\right)\right]\right\}
\end{aligned}
\end{gathered}
$$

for $a, a^{\prime} \in \tilde{\mathfrak{a}}^{\circ}$.

THEOREM 2. Let $X=A / B=E / F$ be a $C$-space where $A, B$ are compact groups, $E, F$ are complex groups. Suppose that $G \rightarrow P \rightarrow X$ is a holomorphic principal fibration with the complex Lie group $G$ as structure group where the action of $E$ on $X$ lifts analytically to action on $P$. Then the complex A-invariant connexions on $P$ are given by the complex linear mappings $\tilde{\chi}: \tilde{\mathfrak{a}}^{\circ} \rightarrow \mathfrak{g}_{h}$ such that

(i) $\tilde{\chi}[a, b]=[\tilde{\chi}(a), \tilde{\chi}(b)]$ for $a \in \tilde{\mathfrak{a}}^{\circ}, b \in \tilde{\mathfrak{b}}^{\circ}$,

(ii) $\tilde{\chi}(f)=\phi_{*}(f)$ whenever $\rho(f) \in \mathfrak{f}_{h}$ where $\rho$ is given by (2.4). The curvature $\tilde{\Xi}$ is assumed of type $(1,1)$ and is given by $\tilde{\Xi}\left(a, \bar{a}^{\prime}\right)=2\left\{\tilde{\chi}\left[a, a^{\prime}\right]-\left[\tilde{\chi}(a), \tilde{\chi}\left(a^{\prime}\right)\right]\right\}$ upon identifying $\tilde{\mathfrak{a}}^{\circ}$ with $\varepsilon_{h}$ by $\rho$.

III. Real and complex torsion on homogeneous complex manifolds. In this section we shall discuss real and complex torsion on homogeneous manifolds. In the first part, a derivation of the expression for the usual (real) torsion is obtained in such a manner that it may formally be repeated in the complex case. The complex torsion is of interest because its vanishing, for metric connexions, is equivalent to the Kähler condition on a complex manifold. Because of this, our expression for the complex torsion, when coupled with the form of a metric connexion to be given in §IV below, will allow us to derive many results which have heretofore had a topological or group-theoretic derivation.

Let $X$ be a manifold, $P(X)$ the bundle of frames on $X$ so that we may write $\mathrm{GL}(n, \mathbf{R}) \rightarrow P(X) \stackrel{\pi}{\rightarrow} X$ where $n=\operatorname{dim}_{\mathbf{R}} X$. The fundamental form $\tilde{\omega}$ on $P(X)$ may be described as follows. Let $V$ be a fixed vector space taken as a standard fibre of $T(X)$, and let $\xi_{1}, \cdots, \xi_{n}$ be a basis of $V$. Then the fibre $\pi^{-1}(x) \subset P(X)$ may be thought of in the following way: each $p \in \pi^{-1}(x)$ is a frame $\left(x, t_{1}, \cdots, t_{n}\right)$ and to $p$ we associate the linear mapping $p: V \rightarrow T_{x}(X)$ defined by $p\left(\xi_{i}\right)=t_{i}$. Then $\tilde{\omega}$ is the $V$-valued form given by

$$
\tilde{\omega}_{p}(t)=p^{-1}\left(\pi_{*}(t)\right) \quad \text { for } t \in T_{p}(P)
$$


Proposition 3.1. Let $\sigma^{*} \tilde{\omega}=\psi$ so that $\psi$ is a V-valued form on $A$. Then $\psi$ is left-invariant on $A$.

(We follow here the notations of $\S \mathrm{I}$.)

Proof. The proof is easy since $\tilde{\omega}$ depends only upon the differentiable structure of $X$.

If we have a linear connexion $\omega$ in $\mathrm{GL}(n, \mathbf{R}) \rightarrow P(X) \stackrel{\pi}{\rightarrow} X, \omega$ has its values in $\operatorname{gl}(V)$ and hence, for $t \in T(P(X)), v \in V, \omega(t) \circ v$ is defined. Matters being so, the torsion form $\zeta$ on $P(X)$ is given by

$$
\zeta\left(t, t^{\prime}\right)=d \tilde{\omega}\left(t, t^{\prime}\right)+\frac{1}{2}\left\{\omega(t) \circ \tilde{\omega}\left(t^{\prime}\right)-\omega\left(t^{\prime}\right) \circ \tilde{\omega}(t)\right\}
$$

for $t, t^{\prime} \in T(P(X))$. Hence, for $a, a^{\prime} \in \mathfrak{a}^{\circ}$, we have that

$$
\begin{aligned}
\left(\sigma^{*} \zeta\right)\left(a, a^{\prime}\right) & =\left(\sigma^{*} d \tilde{\omega}\right)\left(a, a^{\prime}\right)+\frac{1}{2}\left\{\sigma^{*} \omega(a) \circ \sigma^{*} \tilde{\omega}\left(a^{\prime}\right)-\sigma^{*} \omega\left(a^{\prime}\right) \circ \sigma^{*} \tilde{\omega}(a)\right\} \\
& =d \psi\left(a, a^{\prime}\right)+\frac{1}{2}\left\{\chi(a) \circ \psi\left(a^{\prime}\right)-\chi\left(a^{\prime}\right) \circ \psi(a)\right\} \\
& =-\frac{1}{2} \psi\left[a, a^{\prime}\right]+\frac{1}{2}\left\{\chi(a) \circ \psi\left(a^{\prime}\right)-\chi\left(a^{\prime}\right) \circ \psi(a)\right\} .
\end{aligned}
$$

(The last step here follows from (3.1) and the Maurer-Cartan equation on A.) Setting $\sigma^{*} \zeta=\tau$, we have:

Proposition 3.2. The torsion $\tau \in \operatorname{Hom}\left(\mathfrak{a}^{\circ} \otimes \mathfrak{a}^{\circ}, V\right)$ of a linear connexion $\chi$ in $\mathrm{GL}(n, \mathbf{R}) \rightarrow P(A / B) \rightarrow A / B$ is given by

$$
2 \tau\left(a, a^{\prime}\right)=-\psi\left[a, a^{\prime}\right]+\chi(a) \circ \psi\left(a^{\prime}\right)-\chi\left(a^{\prime}\right) \circ \psi(a)
$$

where $\psi$ is defined by Proposition 3.1.

An example here might be instructive. Let $B \rightarrow A \rightarrow A / B$ be the principal group fibration of a reductive homogeneous space $A / B$; then, by definition, $\mathfrak{a}^{\circ}=\mathfrak{b}^{\circ} \oplus \mathfrak{f}^{\circ}$ and Ad $B: \mathfrak{f}^{\circ} \rightarrow \mathfrak{f}^{\circ}$. We then have

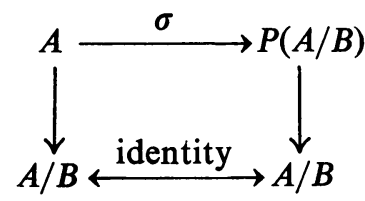

and $A$ is embedded in $P(A / B)$ by $\sigma$. We define an affine connexion in $P(A / B)$ which is in fact a connexion in $A$ (i.e., the horizontal spaces are tangent to $A$ ) by

$$
\begin{array}{ll}
\chi(b)=b, & b \in \mathfrak{b}^{\circ}, \\
\chi(k)=0, & k \in \mathfrak{F}^{\circ} .
\end{array}
$$

The condition (i) on the connexion is the statement of reductiveness (i.e., preservation under infinitesimal right translation by $B$ ). The curvature is given by 


$$
\begin{aligned}
& \Xi\left(a, a^{\prime}\right)=0 \text { if } a \text { or } a^{\prime} \text { lie in } \mathfrak{b}^{\circ}, \\
& \Xi\left(k, k^{\prime}\right)=-\frac{1}{2}\left[k, k^{\prime}\right]_{\mathfrak{b}^{\circ}} .
\end{aligned}
$$

The form $\psi$ is clearly the identity on $\mathfrak{f}^{\circ}$ and 0 on $\mathfrak{b}^{\circ}$; thus the torsion is $2 \tau\left(k, k^{\prime}\right)=\left[k, k^{\prime}\right]_{\boldsymbol{R}^{\circ}}$ and $\tau=0$ if and only if $\left[\mathfrak{f}^{\circ}, \mathfrak{f}^{\circ}\right] \subseteq \mathfrak{b}^{\circ}$ in which case we have a symmetric space. This is the Nomizu canonical affine connexion of the first kind.

Now $\mathfrak{f}^{\circ}=T_{0}(A / B)$ and we may take $V=\mathfrak{f}^{\circ} ; \chi$ should be $\mathrm{gl}(V)$-valued and, in view of reductiveness, this is indeed the case; for $k \in \mathfrak{f}^{\circ}, b \in \mathfrak{b}^{\circ}$, $\chi(b) \circ k=[b, k] \in \mathfrak{f}^{\circ}$. Furthermore, the homomorphism $\phi_{*}: \mathfrak{b}^{\circ} \rightarrow \operatorname{gl}(V)$ is given by $\phi_{*}(b)(k)=[b, k]$; this is just the infinitesimal linear isotropy representation. The verification that $\chi$ satisfies (1.7) is trivial; geometrically, $\mathfrak{f}^{\circ}$ is the left-invariant horizontal space on $A$ and $\mathfrak{b}^{\circ}$ is the vertical space.

Before discussing the complex torsion, it would be well here to give in more detail the structure of a $C$-space $X=A / B$ (compact form) $=E / F$ (complex form). We recall that $\tilde{\mathfrak{a}}^{\circ}=\varepsilon^{\circ}$ and that (2.4) defines an algebra isomorphism between $\varepsilon$ or $\tilde{\mathfrak{a}}^{\circ}$ and $\varepsilon_{\boldsymbol{h}}$. Now $\mathfrak{a}^{\circ} \subset \varepsilon^{\circ}, \mathfrak{b}^{\circ} \subset \mathfrak{f}^{\circ}$ and we may write $\varepsilon^{\circ}=\mathfrak{n}^{\circ} \oplus \mathfrak{f}^{\circ}$ (direct sum of vector spaces) where $\mathfrak{n}^{\circ} \cong T_{0}(X)$ and $\left[\mathfrak{b}^{\circ}, \mathfrak{n}^{\circ}\right] \subseteq \mathfrak{n}^{\circ}$, i.e., the splitting $\varepsilon^{\circ}=\mathfrak{n}^{\circ} \oplus \mathfrak{f}^{\circ}$ may be chosen to be $\mathfrak{b}^{\circ}$-reductive. By complexification, $\tilde{\varepsilon}^{\circ} \cong \varepsilon_{h} \oplus \varepsilon_{h}$ where $\varepsilon_{h}=\mathfrak{n}_{h} \oplus \mathfrak{f}_{h}$ and under the isomorphism (2.4) we may write $\tilde{\mathfrak{a}}^{\circ}=\mathfrak{n}^{*} \oplus \tilde{\mathfrak{f}}$ where $\mathfrak{n}^{*} \cong \mathscr{T}_{0}(X)$ and $\left[\tilde{\mathfrak{b}}^{\circ}, \mathfrak{n}^{*}\right] \subseteq \mathfrak{n}^{*}$. One may describe quite precisely the subalgebras $\tilde{\mathfrak{b}}^{\circ}, \mathfrak{n}^{*}, \mathfrak{f}$, etc., in terms of a Cartan decomposition of $\varepsilon$ into root spaces; this will be done as needed below.

Let $x(t)$ be a smooth curve on a $C^{\infty}$-manifold $X$; in terms of local coordinates $\left(x^{1}, \cdots, x^{n}\right)$, the mapping

$$
t \rightarrow\left(x(t), \frac{\vec{\partial}}{\partial x^{1}}(t), \cdots, \frac{\vec{\partial}}{\partial x^{n}}(t)\right)
$$

gives a local moving frame (cross section of $P(X)$ over $x(t)$ ). The vector tangent to $x(t)$ is given by

$$
\left.\left.\left.\frac{d x(t)}{d t}\right]_{t_{0}}=\sum_{j=1}^{n} \frac{\vec{\partial}}{\partial x^{i}}\right]_{x\left(t_{0}\right)} \frac{d x^{i}}{d t}\right]_{t_{0}}
$$

more generally, for an arbitrary moving frame $\left(x(t), e_{1}(t), \cdots, e_{n}(t)\right)$ with a co-frame $\left(x(t), \omega^{1}(t), \cdots, \omega^{n}(t)\right)$,

i.e.,

$$
\left.\left.\frac{d x(t)}{d t}\right]_{t_{0}}=\sum_{j=1}^{n} \mathbf{e}_{j}\left(t_{0}\right) \frac{\omega^{j}(t)}{d t}\right]_{t_{0}},
$$

$$
d x=\sum_{=1}^{n} \mathbf{e} \omega
$$


A connexion geometrically gives the infinitesimal deviation of each $\mathbf{e}_{i}(t)$ from parallel displacement;

$$
\mathrm{de}_{i}=\sum_{j=1}^{n} \omega_{i}^{j} \mathbf{e}_{j} .
$$

With this in mind, the torsion is the vector form

$$
\begin{aligned}
d(d x) & =\sum_{j=1}^{n} d\left(\mathbf{e}_{j} \otimes \omega_{j}\right) \\
& =\sum_{j, k=1}^{n} \mathbf{e}_{k} \otimes \omega_{j}^{k} \wedge \omega^{j}+\sum_{j=1}^{n} \mathbf{e}_{j} \otimes d \omega_{j} ;
\end{aligned}
$$

for the coordinate frame $e^{j}=\vec{\partial} / \partial x^{j}$, we simply get

$$
d(d x)=\sum_{j, i<k} \frac{\partial}{\partial x^{j}} \otimes\left(\Gamma_{i k}^{j}-\Gamma_{k i}^{j}\right) d x^{i} \wedge d x^{k} .
$$

For an arbitrary frame $(x, e)=\left(x, e_{1}, \cdots, e_{n}\right)$, denoting by $(x, n)$ the natural frame

$$
\left(x, \frac{\vec{\partial}}{\partial x^{1}}, \cdots, \frac{\vec{\partial}}{\partial x^{n}}\right)
$$

$\mathbf{e}(x)=g(x) \mathbf{n}(x)$ where $g(x) \in \mathrm{GL}(n, \mathbf{R})$ and the torsion form is transformed pointwise by $g^{-1}(x)$ on the left.

In the complex analytic case, we may take either real or complex displacements $d x$; the former leads to the torsion of the underlying real connexion, the latter to the complex torsion. Having in mind the latter case, we write locally, for a complex connexion $\Gamma, \theta_{\beta}^{\alpha}=\Gamma_{\beta \tau}^{\alpha} d z^{\tau}$ and

$$
\omega_{\beta}^{\alpha}=Y_{\tau}^{\alpha} d X_{\beta}^{\tau}+Y_{\tau}^{\alpha} \theta_{\gamma}^{\tau} X_{\beta}^{\gamma}
$$

where $X_{\beta}^{\alpha} Y_{\gamma}^{\beta}=\delta_{\gamma}^{\alpha}$ and $\left(z^{1}, \cdots, z^{n}, X_{\beta}^{\alpha} ; \alpha, \beta=1, \cdots, n\right)$ are local coordinates in the complex analytic frame bundle of the complex manifold $X$. Thus

$$
d(d x)=\sum_{\alpha \beta \gamma \tau} \frac{\partial}{\partial z^{\gamma}} \otimes\left(\Gamma_{\beta \tau}^{\alpha}-\Gamma_{\tau \beta}^{\alpha}\right) d z^{\beta} \wedge d z^{\tau}
$$

this is in terms of the coordinate frame; for another frame we operate on the left pointwise by $\operatorname{GL}(n, \mathrm{C})$. Now it is known that an Hermitian metric $\left(h_{\alpha \bar{\beta}}\right)=h$ on $X$ gives a natural complex connexion ${ }^{(3)} \theta=h^{-1} \partial h$ (i.e., $\theta_{\beta}^{\alpha}=h^{\alpha \bar{\tau}} \partial h_{\beta \bar{\tau}}$ ). For this connexion

$$
\Gamma_{\beta \tau}^{\alpha}=h^{\alpha \bar{\gamma}} \frac{h_{\beta \bar{\gamma}}}{\partial z^{\tau}}
$$

and

(3) See Proposition 1 in [7]. 


$$
\begin{aligned}
\left(\Gamma_{\beta \tau}^{\alpha}-\Gamma_{\tau \beta}^{\alpha}\right) & =h^{\alpha \bar{\gamma}} \frac{\partial h_{\beta \bar{\gamma}}}{\partial z^{\tau}}-h^{\alpha \bar{\gamma}} \frac{\partial h_{\tau \bar{\gamma}}}{\partial z^{\beta}} \\
& =h^{\alpha \bar{\gamma}}\left(\frac{\partial h_{\beta \bar{\gamma}}}{\partial z^{\tau}}-\frac{\partial h_{\tau \bar{\gamma}}}{\partial z^{\beta}}\right) .
\end{aligned}
$$

The vanishing of the torsion is thus precisely the Kähler condition on the metric $h$. From this discussion, we see that the complex torsion is a $(1,0)$-vectorial two form of type $(2,0)$ (i.e., a cross section of $\left.\mathscr{T}(X) \otimes \mathscr{T}(X)^{\prime} \wedge \mathscr{T}(X)^{\prime}\right)$ and is obtained from the complex connexion by the same formal operations which one performs on a real connexion to get the real torsion.

Consider now the principal complex analytic fibration $\mathrm{GL}(n, \mathrm{C}) \rightarrow P \rightarrow X$ over a $C$-space $X=A / B=E / F$. Associated to $T(X) \rightarrow X$ we have a standard fibre $V$; then $V \otimes_{\mathrm{R}} \mathrm{C}=V_{h} \oplus V_{\bar{h}}$ so that we have

$$
V_{h} \oplus V_{\bar{h}} \rightarrow \mathscr{T}(X) \oplus \overline{\mathscr{T}(X)}=(T(X))^{\sim} \rightarrow X
$$

as the complexified fibration.

Definition. Set $(\tilde{\psi}(a))^{\sim}=\psi(\rho(a))$ for $a \in \tilde{\mathfrak{a}}^{\circ}$ and also define the complex torsion $\tilde{\tau}$ by $\tilde{\tau}\left(a, a^{\prime}\right)=\tau\left(\rho(a), \rho\left(a^{\prime}\right)\right)$ where $\tau$ was defined in $\S$ III. Note that $\tilde{\tau}$, in contrast to $\tilde{\Xi}$, is a form of type $(2,0)$ and this accounts for the difference in defitions. The following equation justifies our definitions: for $a, a^{\prime} \in \mathfrak{a}^{\circ}$,

$$
\begin{aligned}
\tilde{\tau}\left(a, a^{\prime}\right) & =\tau\left(\rho(a), \rho\left(a^{\prime}\right)\right) \\
& =-\frac{1}{2} \psi\left[\rho(a), \rho\left(a^{\prime}\right)\right]-\frac{1}{2} \chi(\rho(a)) \psi\left(\rho\left(a^{\prime}\right)\right)+\frac{1}{2} \chi\left(\rho\left(a^{\prime}\right)\right) \psi(\rho(a)) \\
& =-\frac{1}{2} \tilde{\psi}\left[a, a^{\prime}\right]-\frac{1}{2} \tilde{\chi}(a) \tilde{\psi}\left(a^{\prime}\right)+\frac{1}{2} \tilde{\chi}\left(a^{\prime}\right) \tilde{\psi}(a)
\end{aligned}
$$

(since $\rho$ is a homomorphism). That is

$$
2 \tilde{\tau}\left(a, a^{\prime}\right)=-\tilde{\psi}\left[a, a^{\prime}\right]-\tilde{\chi}(a) \tilde{\psi}\left(a^{\prime}\right)+\tilde{\chi}\left(a^{\prime}\right) \tilde{\psi}(a), \quad a, a^{\prime} \in \tilde{\mathfrak{a}}^{\circ} .
$$

From (4.1) and from the discussions above, we see that $\tilde{\tau}$ is $V_{h}$-valued ${ }^{(4)}$ and in fact gives precisely the complex torsion as originally defined above. Recalling the structure of a $C$-space $X=A / B=E / F$ as described above, we have the $b^{\circ}$ reductive splitting $\varepsilon_{h}=\mathfrak{n}_{h} \oplus \mathfrak{f}_{h}$ or $\tilde{\mathfrak{a}}^{\circ}=\mathfrak{n}^{*} \oplus \mathfrak{f}$ where $\mathfrak{n}^{*} \cong \mathscr{T}_{0}(A / B)$ and we may take $V_{h}=\mathfrak{n}^{*}$ whereby $\tilde{\psi}$ is simply the projection of $\tilde{\mathfrak{a}}^{\circ}$ on $\mathfrak{n}^{*}$.

IV. Metric geometry of $C$-spaces. We now determine the form of a complex metric connexion on a $C$-space $X$; naturally we desire $A$-invariant metrics. Such an $A$-invariant Hermitian metric is given by a bilinear mapping $Q: \mathfrak{n}^{*} \times \mathfrak{n}^{*} \rightarrow \mathrm{C}$ satisfying

(4) We remark on the obvious fact that $\tilde{\omega}: \mathscr{T}(p) \rightarrow V_{h}$ and $\tilde{\omega}(\bar{v})=\overline{\tilde{\omega}(v)}$ 


$$
Q\left(\alpha n, \beta n^{\prime}\right)=\alpha \bar{\beta} Q\left(n, n^{\prime}\right) \quad \text { for } n, n^{\prime} \in \mathfrak{n}^{*} \text {, }
$$

(ii) $\quad Q\left(\operatorname{Ad} b(n), \operatorname{Ad} b\left(n^{\prime}\right)\right)=Q\left(n, n^{\prime}\right) \quad$ for $b \in B$, or

(ii') $Q\left([b, n], n^{\prime}\right)+Q\left(n,\left[b, n^{\prime}\right]\right)=0$ for $b \in \mathfrak{b}^{\circ}$,

(iii) $\quad Q(n, n)>0$ for $n \in \mathfrak{n}^{*}$.

As mentioned above, for such a $Q$ there is a unique complex connexion $\tilde{\chi}_{Q}$ such that parallel translation preserves the Hermitian inner product. Before isolating $\tilde{\chi}_{Q}$, we must make one comment about covariant differentiation on local sections of a complex analytic vector bundle. Let $M$ be a complex manifold and $B \rightarrow^{\pi} M$ an analytic vector bundle over $M$; for a local $\left(C^{\infty}\right)$ cross section $v$ : $U \subset M \rightarrow \pi^{-1}(U), \bar{\partial} v$ is a well-defined cross section over $U$ of $B \otimes \overline{\mathscr{T}(M)^{\prime}}$. Consequently, in trying to "differentiate" $v(z)$, we need only worry about the $(1,0)$ component of the differentiation operation and this is what a complex connexion in $B$ does. Thus covariant differentiation on vectors is given by $D=D^{\prime}+\bar{\partial}$ where $D^{\prime}=\partial+e(\theta)$ for a complex connexion $\theta$ in $B$. Finally,

$$
\begin{aligned}
D_{z}(v) & =\langle D(v), z\rangle & & \\
& =\left\langle D^{\prime}(v), z\right\rangle & & \text { if } z \text { is of type }(1,0), \\
& =\langle\bar{\partial}(v), z\rangle & & \text { if } z \text { is of type }(0,1) .
\end{aligned}
$$

THEOREM 3. For $x, y, z \in \mathfrak{n}^{*}, \tilde{\chi}_{q}$ is given $b y$

$$
Q\left(\tilde{\chi}_{Q}(z) x, y\right)=-Q\left(x, \bar{\partial}_{\bar{z}} y\right)
$$

where $\bar{\partial}_{\bar{z}}(y)=\langle\bar{\partial}(y), \bar{z}\rangle$.

NoTE. As will be seen later, the computation of $\bar{\partial}(y)$ is easily done within the framework of Lie-algebra cohomology.

Proof. Let $\operatorname{dim} A / B=m$ and let $n_{1}, \cdots, n_{m}$ be a basis of $\mathrm{n}^{*}$; then, by left translation, we get a complex frame $\left(e, n_{1}(e), \cdots, n_{m}(e)\right)(e \in E)$ on $E$ which locally around the origin projects onto a complex frame $\left([e], \pi * n_{1}(e), \cdots, \pi * n_{m}(e)\right)=$ $\left(z, n_{1}^{*}(z), \cdots, n_{m}^{*}(z)\right)$ on $X$; this is seen by using canonical coordinates of the first kind in a fashion similar to $[15, \mathrm{pp} .42-43]$. Let $x, y, z$ be vectors in $\mathfrak{n}^{*}=\mathscr{T}_{0}(A / B)$ and let $\tilde{x}(z), \tilde{y}(z), \tilde{z}(z)$ be the unique vectors obtained locally by parallel translation along a geodesic curve $z(t)$ where

Then

$$
\left.\frac{d z(t)}{d t}\right]_{t=t_{0}}=\tilde{z}\left(t_{0}\right)
$$

$$
\begin{aligned}
& x(z)=\sum_{i=1}^{m} \phi_{i}(z) n_{i}^{*}(z), \\
& y(z)=\sum_{j=1}^{m} \eta_{j}(z) n_{j}^{*}(z),
\end{aligned}
$$


where $\phi_{i}, \eta_{j}$ are $C^{\infty}$ complex-valued functions. They may be assumed holomorphic if and only if the connexion is holomorphic (which means that it is flat!). Recalling that if $h(z)=\left(h_{\alpha \bar{\beta}}(z)\right)$ is the matrix of the Hermitian metric, $Q(\tilde{x}(z), \tilde{y}(z))={ }^{t}(\tilde{y}(z) h(z) \tilde{x}(z))$, we have

$$
Q(\tilde{x}(z), \tilde{y}(z))=\sum_{i, j=1}^{m} \phi_{i}(z) \overline{\eta_{j}(z)} Q\left(n_{i}^{*}(z), \eta_{j}^{*}(z)\right)=Q(x, y)
$$

(since parallel translation preserves the Hermitian inner product). But $Q\left(n_{i}^{*}(z), n_{j}^{*}(z)\right)=Q\left(n_{i}, n_{j}\right)$ since the metric is $A$-invariant and thus

$$
\begin{aligned}
\sum_{i, j=1}^{m} \phi_{i}(0) \overline{\eta_{j}(0)} Q\left(n_{i}, n_{j}\right) & =Q(\tilde{x}(z), \tilde{y}(z)) \\
& =\sum_{i, j=1}^{m} \phi_{i}(z) \overline{\eta_{j}(z)} Q\left(n_{i}, n_{j}\right) .
\end{aligned}
$$

We now apply the operation $\tilde{z}(z)]_{0}$ to both ends of this scalar equation:

$$
\left.\left.\sum_{i, j=1}^{m}\left\{\tilde{z}\left(\phi_{i}\right)\right]_{0} \overline{\eta_{j}(0)}+\phi_{i}(0) \tilde{z} \overline{\left(\eta_{j}\right)}\right]_{0}\right\} Q\left(n_{i}, n_{j}\right)=0 .
$$

Now recalling

(i) that the above is taking place along the integral curve $(z(t))$,

(ii) the geometric definition of covariant differentiation (Nomizu [16, p. 52]), and

(iii) the above remarks about complex covariant differentiation (for which the geometrical interpretation is still true), we see that

$$
\begin{aligned}
& \left.\left.\sum_{i=1}^{m} \tilde{z}\left(\phi_{i}\right)\right]_{0} n_{i}=-D_{\tilde{z}}(\tilde{x})\right]_{0}=-D_{\tilde{z}}^{\prime}(\tilde{x})=\tilde{\chi}_{Q}(z) \circ x, \\
& \left.\left.\sum_{j=1}^{m} \tilde{z}\left(\eta_{j}\right)\right]_{0} \bar{n}_{j}=-\partial_{\tilde{z}}(\tilde{y})\right]_{0}=-\overline{\bar{\partial} \tilde{z}-(\tilde{y})]_{0}}=-\overline{\bar{\partial}_{\bar{z}}(y)} .
\end{aligned}
$$

Combining all, we have that $Q\left(\tilde{\chi}_{Q}(z) \circ x, y\right)+Q\left(x, \bar{\partial}_{\bar{z}}(y)\right)=0$ which was to be proven.

Note. The above demonstration was suggested by the proof of the similar Proposition 13.1 in [15]. One might also derive this result using a theorem of Singer [17] which gives the horizontal space explicitly in the Hermitian case. Indeed, a proof using Singer's result would be more in the spirit of the present work. We were not able to find such; however, it perhaps would be of interest to do so.

V. Some applications of the theory. Before continuing on, we shall show how the above theory gives some known results in a fairly explicit manner. We have proven the first part of 
THEOREM 4. The complex torsion of a complex connexion $\tilde{\chi}$ on a $C$-space $A / B=E / F$ is given as a mapping $\tilde{\tau}: \mathfrak{n}^{*} \times \mathfrak{n}^{*} \rightarrow \mathfrak{n}^{*}$ given by $2 \tilde{\tau}\left(n, n^{\prime}\right)=-\left[n, n^{\prime}\right]$ $-\tilde{\chi}(n) \circ n^{\prime}+\tilde{\chi}\left(n^{\prime}\right) \circ n, n, n^{\prime} \in \mathfrak{n}^{*}$. If we have an A-invariant Hermitian metric $Q: \mathrm{n}^{*} \times \mathrm{n}^{*} \rightarrow \mathbf{C}$, then the associated complex connexion $\tilde{\chi}_{Q}$ is given by $Q\left(\tilde{\chi}_{Q}(n) \circ n^{\prime}, n^{\prime \prime}\right)=-Q\left(n^{\prime}, \bar{\partial}_{\bar{n}}\left(n^{\prime \prime}\right)\right), n, n^{\prime}, n^{\prime \prime} \in \mathfrak{\pi}$. The Cartan-Killing form gives a Kähler geometry on $A / B$ if and only if $A / B$ is Hermitian symmetric. There is a natural Kähler metric on a Kähler $C$-space $X$ such that $X$ becomes $a$ simply-connected Einstein space and is in fact an algebraic variety with a negative canonical bundle.

To prove the statement beginning with "The Cartan-Killing form ...,", we shall need to know more precisely the structure of $C$-spaces and a little bit of the structure theory of complex semi-simple Lie algebras. Both of these are reviewed in [4] and we only list the facts we shall use. Upon choosing a Cartan subalgebra $\mathfrak{h} \subset \boldsymbol{\varepsilon}$, we may write

$$
\boldsymbol{\varepsilon}=\mathfrak{h} \oplus\left(\underset{\alpha \in \Sigma}{\oplus} v_{\alpha}\right)
$$

where $\Sigma$ is the root system and $v_{\alpha}$ is a 1-dimensional eigenspace for the root $\alpha$. In particular, we may choose eigenvectors $e_{\alpha} \in v_{\alpha}$ such that $\left[e_{\alpha}, e_{\beta}\right]=N_{\alpha, \beta} e_{\alpha+\beta}(\alpha \neq-\beta)$ where the $N_{\alpha, \beta}$ obey the structure relations given, for example, in [9]. We denote the Cartan-Killing form on $\mathfrak{h}$ by $Q$. The most general $C$-space is given as $X=A / B$ (compact form) $=E / F$ (complex form) where $\boldsymbol{\varepsilon}=\tilde{\mathfrak{a}}^{\circ}$ and we shall describe $B$ and $F$ by describing $\tilde{\mathfrak{b}}^{\circ}$ and $\mathfrak{f}$ (because of Lemma 2.1). Associated to $\tilde{b}^{\circ}$, there is first of all a closed subsystem $\Psi \subset \Sigma$ ( $\Psi$ are the roots of $\left.\tilde{\mathfrak{b}}^{\circ}\right)$. We set $\mathfrak{b}_{\Psi}=$ complex Lie algebra generated by $e_{\alpha}(\alpha \in \Psi)$. Define $\mathfrak{h}_{\Psi}=\{h \in \mathfrak{h},\langle\alpha, h\rangle=0$ for all $\alpha \in \Psi\}$. Then there is a rational splitting $\mathfrak{h}_{\Psi}=\mathfrak{h}_{B} \oplus \mathfrak{h}_{[}$ and a splitting of $\mathfrak{h}_{[}$into complex subspaces: $\mathfrak{h}_{[}=\mathfrak{p} \oplus \overline{\mathfrak{p}}$ such that

(i) $\tilde{\mathfrak{b}}^{\circ}=\mathfrak{h}_{B} \oplus \mathfrak{b}_{\Psi}$,

(ii) $\mathfrak{f}=\tilde{\mathfrak{b}}^{\circ} \oplus \overline{\mathfrak{p}} \oplus \mathfrak{n}$ where $\mathfrak{n}={ }_{\mathrm{c}}\left(e_{\alpha}: \alpha \in \Sigma^{-}-\Psi^{-}\right)\left(^{-}\right.$denotes negative roots relative to an ordering). We observe three things:

(i) $^{\prime}$ If $\mathfrak{n}^{*}={ }_{\mathrm{c}}\left(e_{\alpha}: \alpha \in \Sigma^{+}-\Psi^{+}\right)$, this $\mathfrak{n}^{*}$ is the same as $\mathfrak{n}^{*}$ above; $\mathfrak{n}^{*} \cong \mathscr{T}_{0}(A / B)$, whenever $\mathfrak{p}=0$. If $\mathfrak{p} \neq 0, \mathscr{T}_{0}(A / B) \cong \mathfrak{n}^{*} \oplus \mathfrak{p}$.

(ii)' $\overline{\mathfrak{p}} \oplus \mathfrak{h}_{B} \oplus\left(\dot{\mathfrak{b}}_{\Psi} \cap \mathfrak{h}\right)$ lies on no rational hyperplane.

This section is concerned with those $C$-spaces $X=A / B=E / F$ such that $\mathfrak{p}=0$. In this case, $\mathfrak{n}^{*} \cong \mathscr{T}_{0}(A / B)$ and $\mathfrak{n} \cong \overline{\mathscr{T}}_{0}(A / B)$. Thus it makes sense to speak of $\bar{\partial}_{\bar{z}}(y)\left(y, z \in \mathfrak{n}^{*}\right)$ and the final remark is that

(iii) $\bar{\partial}_{\bar{z}}(y)=[\bar{z}, y]_{\mathfrak{n}^{*}}$ (conjugation with respect to the compact form $A$ of $E$ ). Thus if $y=e_{\beta}, z=e_{\alpha}\left(\alpha, \beta \in \Sigma^{+}-\Psi^{+}\right)$,

$$
\bar{\partial}_{\bar{e}_{\alpha}}\left(e_{\beta}\right)=\bar{\partial}_{e_{-x}}\left(e_{\beta}\right)=\left[e_{-\alpha}, e_{\beta}\right]=N_{-\alpha+\beta}^{\prime} e_{\alpha+\beta}
$$

where 


$$
\begin{aligned}
& N_{-\alpha, \beta}^{\prime}=N_{-\alpha, \beta} \quad\left(-\alpha+\beta \in \Sigma^{+}-\Psi^{+}\right), \\
& N_{-\alpha, \beta}^{\prime}=0 \quad \text { otherwise. }
\end{aligned}
$$

Let $Q=$ Cartan-Killing form on $\mathfrak{n}^{*}$ and $\tilde{\chi}_{Q}=\chi$; then, by (5.1),

$$
Q\left(\chi\left(e_{\alpha}\right) e_{\gamma}, e_{\beta}\right)=Q\left(e_{\gamma}, N_{\beta .-\alpha}^{\prime} e_{\beta-\alpha}\right) \quad\left(N_{-\alpha, \beta}^{\prime}=-N_{\beta,-\alpha}{ }^{\prime}\right) .
$$

On the other hand, if $\tilde{\tau}_{Q}=\tau, 2 \tau\left(e_{\alpha}, e_{\beta}\right)=-N_{\alpha, \beta} e_{\alpha+\beta}-\chi\left(e_{\alpha}\right) e_{\beta}+\chi\left(e_{\beta}\right) e_{\alpha}$. Combining,

$$
\begin{aligned}
2 Q\left(\tau\left(e_{\alpha}, e_{\beta}\right), e_{\gamma}\right) & =Q\left(-N_{\alpha, \beta} e_{\alpha+\beta}-\chi\left(e_{\alpha}\right) e_{\beta}+\chi\left(e_{\beta}\right) e_{\alpha}, e_{\gamma}\right) \\
& =-N_{\alpha, \beta} \delta_{\alpha+\beta}^{\gamma}+N_{\gamma,-\alpha} \delta_{\gamma-\alpha}^{\beta}-N_{\gamma,-\beta} \delta_{\gamma-\beta}^{\alpha} \\
& =0 \quad \alpha+\beta-\gamma \neq 0 \\
& =-N_{\alpha, \beta}+N_{\gamma,-\alpha}-N_{\gamma,-\beta} \quad \alpha+\beta-\gamma=0 .
\end{aligned}
$$

Assume that $\alpha+\beta-\gamma=0, \alpha+\beta \neq 0, \beta-\gamma \neq 0, \alpha-\gamma \neq 0$. Then

and

$$
N_{\alpha, \beta}=N_{\beta,-\gamma}=N_{-\gamma, \alpha}=-N_{\gamma,-\beta}
$$

$$
2 Q\left(\tau\left(e_{\alpha,}, e_{\beta}\right), e_{\gamma}\right)=-N_{\alpha, \beta}-N_{\alpha, \beta}-N_{\alpha, \beta}=-3 N_{\alpha, \beta} .
$$

By arguing out the low dimensional cases to justify the assumption, we get

Proposition 5.1. $\tau=0$ for the Cartan-Killing form if and only if $\mathrm{n}^{*}$ is a commutative Lie algebra which happens if and only if $A / B$ is Hermitiansymmetric. That is, the Cartan-Killing form gives an Hermitian geometry on a Kähler $C$-space $A / B$ which is a Kähler geometry if and only if $A / B$ is Hermitian-symmetric.

The above is similar to the main theorem of Hano and Matsushima [10]. An affine connexion $\chi$ on a reductive homogeneous space $A / B\left(\mathfrak{a}^{\circ}=\mathfrak{b}^{\circ} \oplus \mathfrak{f}^{\circ}\right)$ is said to be of the first kind if $\chi(k) \circ k^{\prime}=\frac{1}{2}\left[k, k^{\prime}\right]_{t^{\circ}}$. The result of Hano and Matsushima states that, for a complex metric connexion $\tilde{\chi}_{Q}$ where $Q$ is Kähler, $\tilde{\chi}_{Q}$ is of the first kind for $A / B$ if and only if $A / B$ is Hermitian-symmetric. Recalling that the complex Hermitian geometry coincides with the underlying Riemannian geometry if and only if the Hermitian connexion is torsion-free and that the Kähler condition implies that $\tilde{\chi}_{Q}(x) \circ J_{X}(y)=J_{X}\left(\tilde{\chi}_{Q}(x) \circ y\right)$ for $x, y \in n^{*}$, a calculation similar to the above gives

Proposition 5.1'. Let $A / B$ be a $C$-space with $\mathfrak{p}=0$ as described above. In order that a Riemannian connexion on $A / B$ induced by an invariant Kähler metric be of the first kind, it is necessary and sufficient that $A / B$ be Hermitiansymmetric. 
Having seen what metric geometries on $A / B(\mathfrak{p}=0)$ are not Kähler, we ask what (if any) metrics give a torsion-free connexion. Choose a linear form $\eta \in \mathfrak{h}^{\prime}$ such that $\langle\eta, h\rangle=0$ for all $h \in \mathfrak{h} \cap \mathfrak{b}_{\Psi}$. We define an Ad $B$-invariant inner product $Q_{\eta}$ on $n^{*}$ by

$$
Q_{\eta}\left(e_{\alpha}, e_{\beta}\right)=\delta_{\beta}^{\alpha} Q(\alpha, \eta) .
$$

Then, such an $\eta$ defines an (in general degenerate) invariant Kähler geometry on $A / B$. Indeed, if $\tau=\tau_{Q_{n}}, \chi_{n}=\chi_{Q_{n}}$,

$$
\begin{aligned}
Q_{\eta}\left(\tau\left(e_{\alpha}, e_{\beta}\right), e_{\gamma}\right) & =Q_{\eta}\left(-N_{\alpha, \beta} e_{\alpha+\beta}-\chi_{\eta}\left(e_{\alpha}\right) e_{\beta}+\chi_{\eta}\left(e_{\beta}\right) e_{\alpha}, e_{\gamma}\right) \\
& \left.=-N_{\alpha, \beta}(\eta, \gamma)+N_{\gamma,-\alpha}(\eta, \beta)-N_{\gamma,-\beta}(\eta, \alpha) \quad \text { (assuming } \alpha+\beta=\gamma\right) \\
& =-N_{\alpha, \beta}(\eta, \gamma-\beta-\alpha) \\
& =0 .
\end{aligned}
$$

There is a canonical element $g \in \mathfrak{h}^{\prime}, g=\frac{1}{2} \sum_{\alpha \in \Sigma^{+}} \alpha ; g$ is canonical for $\tilde{\mathfrak{a}}^{\circ}$; for $\tilde{b}^{\circ}$, the element $g_{1}=\frac{1}{2} \Sigma_{\alpha \in \Psi+\alpha}$ is canonical. If $g=g_{1}+g_{2}$, then

(i) $g_{2}$ is orthogonal to $\left(\mathfrak{h} \cap \mathfrak{b}_{\Psi}\right)$ and

(ii) $Q\left(g_{2}, \alpha\right) \geqq Q(\alpha, \alpha)$ (for $\alpha \in \Sigma^{+}-\Psi^{+}$). Since the condition that $Q_{\eta}$ constructed above be positive-definite is that $Q(\alpha, \eta)>0$ for $\alpha \in \Sigma^{+}-\Psi^{+}, \eta=g_{2}$ gives an invariant Kähler metric on $A / B$.

This particular Kähler metric $\eta=g_{2}$ has a very nice geometric interpretation as follows. For a Kähler manifold, one has the curvature tensor as a mapping $R: \mathscr{T} \otimes \overline{\mathscr{T}} \rightarrow \operatorname{Hom}(\mathscr{T}, \mathscr{T})$, i.e., $R \in \operatorname{Hom}(\mathscr{T} \otimes \bar{T}, \operatorname{Hom}(\mathscr{T}, \mathscr{T}))$ and for $t, t^{\prime} \in \mathscr{T}$, one defines the Ricci form $\widetilde{R}$ by $\widetilde{R}\left(t, \tilde{t}^{\prime}\right)=\operatorname{tr} R\left(t, \tilde{t}^{\prime}\right) ; \widetilde{R} \in \operatorname{Hom}(\mathscr{T} \otimes \bar{T}, \mathbf{C})$. If, in local coordinates, $R_{\beta \bar{\gamma} \tau}^{\alpha}$ is the curvature tensor, then

$$
R_{\beta \bar{\gamma}}=\sum_{\tau=1}^{m} R_{\beta \bar{\gamma} \tau}^{\tau} \text {. }
$$

Now one knows furthermore that

$$
-\frac{1}{2 \pi i} \sum_{\alpha, \beta=1}^{m} R_{\alpha \beta} d z^{\alpha} \wedge d \bar{z}^{\beta}
$$

gives the first Chern class of the compact Kähler manifold in question.

Proposition 5.2. The $C$-spaces $A / B=E / F$ with $\mathfrak{p}=0$ as given above are all Kähler-Einstein spaces with the Kähler metric given by $Q_{g_{2}}$.

That the Kähler $C$-spaces are Einstein spaces may be found in [13].

Proof. We must compute the trace of the linear mapping $\tilde{\Xi}\left(e_{\alpha}, e_{-\beta}\right): \mathfrak{n}^{*} \rightarrow n^{*}$ given by $\tilde{\Xi}\left(e_{\alpha}, e_{-\beta}\right) e_{\gamma}=\frac{1}{2}\left\{\left[\chi\left(e_{\alpha}\right), \chi\left(e_{-\beta}\right)\right] e_{\gamma}-\chi\left[e_{\alpha}, e_{-\beta}\right] e_{\gamma}\right\}$ where $\chi$ is the connexion associated to the Hermitian metric described in the proposition. Thus $\tilde{\Xi}\left(e_{\alpha}, e_{-\beta}\right) e_{\gamma}=A_{1} e_{\gamma}+A_{2} e_{\gamma}$, where $A_{1} e_{\gamma}=\frac{1}{2}\left[\chi\left(e_{\alpha}\right), \chi\left(e_{-\beta}\right)\right] e_{\gamma}$ and $A_{2} e_{\gamma}=-\frac{1}{2} \chi\left[e_{\alpha}, e_{-\beta}\right] e_{\gamma}$. Since $\operatorname{tr}\left(A_{1}+A_{2}\right)=\operatorname{tr} A_{1}+\operatorname{tr} A_{2}$ and $\operatorname{tr} A_{1}=0\left(A_{1}\right.$ is a commutator $)$, we con- 
clude that $\operatorname{tr} \tilde{\Xi}\left(e_{\alpha}, e_{-\beta}\right)=-\frac{1}{2} \operatorname{tr} \chi\left[e_{\alpha}, e_{-\beta}\right]$. But

$$
Q_{g_{2}}\left(\chi\left[e_{\alpha}, e_{-\beta}\right] e_{\tau}, e_{\tau}\right)=N_{\alpha,-\beta} Q_{g_{2}}\left(\chi\left(e_{\alpha-\beta}\right) e_{\tau}, e_{\tau}\right)=0
$$

unless $\alpha=\beta$ in which case

$$
Q_{g_{2}}\left(\left[e_{\alpha}, e_{-\alpha}\right] e_{\tau}, e_{\tau}\right)=Q_{g_{2}}\left(\left[h_{\alpha}, e_{\tau}\right], e_{\tau}\right)=Q(\alpha, \tau) Q\left(g_{2}, \tau\right) .
$$

On the other hand, $Q_{g_{2}}\left(\chi\left[e_{\alpha}, e_{-\beta}\right] e_{\tau}, e_{\tau}\right)=\left(A_{2}\right)_{\alpha-\beta \tau}^{\tau} Q\left(g_{2}, \tau\right)$ which implies that $\left(A_{2}\right)_{\alpha-\beta \tau}^{\tau}=\frac{1}{2} \delta_{\beta}^{\alpha} Q(\alpha, \tau)$

$$
R_{\alpha \bar{\beta}}=\delta_{\beta}^{\alpha \frac{1}{2}} \sum_{\tau} Q(\alpha, \tau)=\delta_{\beta}^{\alpha} Q\left(g_{2}, \beta\right) .
$$

Q.E.D.

Corollary 1 (Borel-Hirzebruch[3]). In a $C$-space $A / B$ with $\mathfrak{p}=0$, the canonical bundle of $A / B$ is a negative line bundle [12] and thus these $C$-spaces are algebraic varieties.

Corollary 2 (Borel-Goto). A C-space $A / B$ with $\mathfrak{p}=0$ is simply connected.

Proof. Follows from [10].

This corollary is definitely not true for a general $C$-space.

VI. Canonical geometries in homogeneous vector bundles. In the preceding section we derived geometric properties of those $C$-spaces $A / B$ (compact form) where $\operatorname{rank} B=\operatorname{rank} A(\Leftrightarrow \mathfrak{p}=0)$. We now relax this restriction. In $T(X)$ where $X=A / B=E / F$, the $A$-invariant complex connexions are given by the linear mappings $\tilde{\chi}: \varepsilon=\tilde{\mathfrak{a}}^{\circ} \rightarrow \operatorname{gl}(n, \mathbf{C})\left(n=\operatorname{dim}_{\mathbf{C}} X\right)$ such that

$$
\begin{aligned}
\tilde{\chi}[e, b] & =[\tilde{\chi}(e), \tilde{\chi}(b)], \quad e \in \varepsilon, \quad b \in \tilde{\mathfrak{b}}^{\circ}, \\
\tilde{\chi}(f) & =\operatorname{ad} f: \varepsilon / \mathfrak{f}: \rightarrow \varepsilon / \mathfrak{f}, \quad f \in \mathfrak{f} .
\end{aligned}
$$

Definition 6.1. Let $\rho: F \rightarrow \mathrm{GL}\left(V^{\rho}\right)$ be a holomorphic representation of $F$ on a finite-dimensional $\mathrm{C}$-module $V^{\rho}$. Associated to the fibering $F \rightarrow E \rightarrow F / E$, we form the holomorphic vector bundle $V^{\rho} \rightarrow \mathrm{V}^{\rho}=E \times{ }_{F} V^{\rho} \rightarrow E / F$. Then $\mathrm{V}^{\rho}$ is by definition a homogeneous vector bundle. We observe that $\mathscr{T}(X)=E \times{ }_{F} \varepsilon / \mathfrak{f}$ where $F$ acts on $\varepsilon / \mathfrak{f}$ by "Ad.",

Proposition 6.1. Define $\tilde{\chi}$ by

$$
\begin{aligned}
& \tilde{\chi}(n)=0 \quad\left(n \in \mathfrak{n}^{*} \oplus \mathfrak{p}\right), \\
& \tilde{\chi}(f)=\text { ad } \quad f: \varepsilon / \mathfrak{f} \rightarrow \varepsilon / \mathfrak{f} \quad(f \in \mathfrak{f}) .
\end{aligned}
$$

Then $\tilde{\chi}$ gives a complex connexion in $\mathscr{T}(X)$ considered as a homogeneous vector bundle.

Proof. Since $\left[\mathfrak{n}^{*}, \tilde{\mathfrak{b}}^{\circ}\right] \subseteq \mathfrak{n}^{*}$ and $\left[\mathfrak{p}, \tilde{\mathfrak{b}}^{\circ}\right]=0$, one easily checks that (i) and (ii) above are satisfied. Q.E.D. 
This connexion is actually the connexion in $F \rightarrow E \rightarrow E / F$ which is the complex analogue of the example discussed in §III.

Definition 6.2. The above connexion is called the canonical complex affine connexion on $E / F$.

Proposition 6.2. The canonical complex affine connexion in $\mathscr{T}(X)$ gives a metric connexion $\Leftrightarrow E / F$ is Hermitian symmetric.

Proof. Suppose that $\tilde{\chi}=\chi_{Q}$ for some metric $Q$ ( $Q$ need not be the CartanKilling form). Then, for $n, n^{\prime}, n^{\prime \prime} \in \mathfrak{n}^{*} \oplus \mathfrak{p}, 0=Q\left(\chi_{Q}(n) \circ n^{\prime}, n^{\prime \prime}\right)=-Q\left(n^{\prime}, \bar{\partial}_{\bar{n}}\left(n^{\prime \prime}\right)\right)$ $= \pm Q\left(n^{\prime},\left[\bar{n}, n^{\prime \prime}\right]_{\mathfrak{n}^{*} \oplus \mathfrak{p}}\right)$. It follows immediately that $\mathfrak{p}=0$, i.e., $\operatorname{rank} B=\operatorname{rank} A$. If there exists $\alpha, \beta \in \Sigma^{+}-\Psi^{+}$such that $\alpha+\beta \in \Sigma^{+}-\Psi^{+}$, then $-\beta \in \Sigma^{-}-\Psi^{-}$ and $0=Q\left(e_{\beta},\left[e_{-\beta}, e_{\alpha+\beta}\right]\right)=N_{-\beta, \alpha+\beta} Q\left(e_{\beta}, e_{\beta}\right) \neq 0$; a contradiction which implies that $n^{*}$ is commutative. Q.E.D.

The canonical complex connexion is in the fibration $F \rightarrow E \rightarrow E / F$; its curvature form is an $\mathfrak{f}$-valued form of type $(1,1)$ (recall Lemma 2.2 ) and is given by

$$
\tilde{\Xi}\left(n, \bar{n}^{\prime}\right)=-\frac{1}{2}\left[n, \bar{n}^{\prime}\right]_{\mathfrak{f}} \quad\left(n, n^{\prime} \in \mathfrak{n}^{*} \oplus \mathfrak{p}\right) .
$$

If $\rho: F \rightarrow F^{\prime}$ is a holomorphic homomorphism of complex Lie groups, the associated to the fibering $F \rightarrow E \rightarrow E / F$, we have $F^{\prime} \rightarrow E \times{ }_{F} F^{\prime} \rightarrow E / F$ and $\tilde{\chi}$ goes over into a complex connexion $\tilde{\chi}^{\prime}$. The curvature of $\tilde{\chi}^{\prime}$ in $F^{\prime} \rightarrow E \times{ }_{F} F^{\prime} \rightarrow E / F$ is $\mathfrak{f}^{\prime}$-valued and is given by

$$
\tilde{\Xi}^{\prime}\left(n, \bar{n}^{\prime}\right)=-\frac{1}{2} \rho\left(\left[n, \bar{n}^{\prime}\right]_{\mathfrak{f}} \quad\left(n, n^{\prime} \in \mathfrak{n}^{*} \oplus \mathfrak{p}\right) .\right.
$$

We close this section with two applications to metric geometry.

Proposition 6.3. Let $A / B=E / F$ be a $C$-space with $\mathfrak{p} \neq 0$. Then for no $A$-invariant Hermitian metric in $\mathscr{T}(A / B)$ can $\tilde{\tau}_{Q}=0$ where $\tilde{\tau}_{Q}$ is the complex torsion.

REMARK. Thus $\operatorname{rank} B=\operatorname{rank} A$ is a necessary and sufficient condition that a $C$-space $A / B$ be Kähler. This result is due to Borel and Lichnerowicz and heretofore has had a topological proof.

Proof. Suppose that $Q$ exists with the associated $\tilde{\chi}_{Q}$ and $\tau_{Q}=0$. Then

$$
Q\left(\tilde{\tau}_{Q}\left(n, n^{\prime}\right), n^{\prime \prime}\right)=Q\left(-\left[n, n^{\prime}\right], n^{\prime \prime}\right)-Q\left(\tilde{\chi}_{Q}(n), n^{\prime}, n^{\prime \prime}\right)+Q\left(\tilde{\chi}_{Q}\left(n^{\prime}\right), n, n^{\prime \prime}\right)
$$

or

$$
0=-Q\left(\left[n, n^{\prime}\right], n^{\prime \prime}\right)+Q\left(n^{\prime},\left[\bar{n}, n^{\prime \prime}\right]_{\mathfrak{n} * \oplus \mathfrak{p}}\right)-Q\left(n,\left[\bar{n}^{\prime}, n^{\prime \prime}\right]_{\mathfrak{n}^{* \oplus \mathfrak{p}}}\right) .
$$

Set $n=e_{\alpha}, n^{\prime}=k \in \mathfrak{p}, n^{\prime \prime}=e_{\alpha}$; then

$$
\begin{aligned}
0 & =Q\left(\left[e_{\alpha}, k\right], e_{\alpha}\right)+Q\left(k,\left[e_{-\alpha}, e_{\alpha}\right]_{\mathfrak{n} * \oplus \mathfrak{p}}\right)+Q\left(e_{\alpha},\langle\bar{k}, \alpha\rangle e_{\alpha}\right) \\
& =-\langle\alpha, k\rangle Q\left(e_{\alpha}, e_{\alpha}\right)+Q\left(k,\left(h_{\alpha}\right)_{\mathfrak{n} * \oplus \mathfrak{p}}\right)+\langle\alpha, k\rangle Q\left(e_{\alpha}, e_{\alpha}\right)
\end{aligned}
$$


and $Q\left(k,\left(h_{\alpha}\right)_{\mathfrak{n} * \oplus_{\mathfrak{p}}}\right)=0$. But since $\mathfrak{p} \neq 0$ and $\mathfrak{f} \cap \mathfrak{h}$ lies on no rational hyperplane, we may find an $\alpha$ such that $\left(h_{\alpha}\right)_{\mathfrak{n}^{*} \oplus \mathfrak{p}} \neq 0$; for this $\alpha$ we take $k=\left(h_{\alpha}\right)_{\mathfrak{n}^{*} \oplus \mathfrak{p}}$ $\left(\left(h_{\alpha}\right)_{\mathfrak{n}^{*} \oplus \mathfrak{p}} \subseteq \mathfrak{p}\right)$ and have $Q(k, k)=0, k \neq 0$ which is a contradiction. Q.E.D.

Now Proposition 6.2. shows that the canonical complex connexion in a homogeneous vector bundle $V^{\rho} \rightarrow \mathrm{V}^{\rho} \rightarrow E / F$ is not in general a metric connexion. For the purposes of harmonic theory and vanishing theorems, a metric connexion is required. On the other hand, the curvature of the canonical complex connexion is easily computable. The gap is bridged for line bundles by

Proposition 6.4. Suppose that $\rho$ is 1-dimensional and $A / B$ is Kähler. Then if $\tilde{\chi}_{\rho}$ is the canonical complex connexion in $V^{\rho} \rightarrow V^{\rho} \rightarrow A / B, \tilde{\chi}_{\rho}$ may be taken to be a metric connexion for an Hermitian metric $h$ in $\mathrm{V}^{\rho}$; $\tilde{\chi}_{\rho}=\left(h^{-1} \partial h\right)$, and $\tilde{\Xi}_{\rho}=\bar{\partial}\left(h^{-1} \partial h\right)$ is then suitable for harmonic theory.

Proof. For the canonical bundle, Proposition 5.2 gives the result. In the general case, one must parallel the proof of Proposition 2.8 and we omit the calculation.

VII. A geometric realization of the Atiyah construction. In this section we give a geometric interpretation to the curvature class as constructed by Atiyah in [1]. The construction in [1] goes briefly as follows: Let $E \rightarrow P \rightarrow X$ be a complex principal fibration over a complex manifold $X$ with a complex group $E$ as structure group. Since $E$ acts analytically on $P$ on the right, we may form $\mathscr{T}(P) / E$ and in fact have

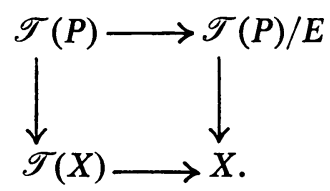

The geometrical interpretation of $\mathscr{T}(P) / E$ is that vectors in $\mathscr{T}(P)$ related by right action of $E$ are identified; the standard fibre of $\mathscr{T}(P) / E$ is $V_{h} \oplus \varepsilon_{h}$ where $V_{h}$ is a standard fibre of $\mathscr{T}(X)$. Setting $Q(P)=\mathscr{T}(P) / E$, we form the Atiyah sequence

$$
0 \rightarrow \mathbf{L}(P) \rightarrow \mathbf{Q}(P) \rightarrow \mathscr{T}(X) \rightarrow 0
$$

where the standard fibre of $\mathbf{L}(P)$ is $\varepsilon_{h}$.

$A C^{\infty}$-splitting of (7.1) always exists and indeed gives a connexion as is clear from the geometrical interpretation. However, an analytic splitting does not in general exist, the obstruction to such is now obtained. Writing $\mathbf{L}=\mathbf{L}(P)$, $\mathbf{Q}=\mathbf{Q}(P), \mathscr{T}=\mathscr{T}(X)$, we consider the derived exact sequence of analytic vector bundles

$$
0 \rightarrow \operatorname{Hom}(\mathscr{T}, \mathbf{L}) \rightarrow \operatorname{Hom}(\mathbf{Q}, \mathbf{L}) \rightarrow \operatorname{Hom}(\mathbf{L}, \mathbf{L}) \rightarrow 0 \text {. }
$$

This gives an exact sequence of sheaves 


$$
0 \rightarrow \operatorname{Hom}(\mathscr{T}, \mathbf{L}) \rightarrow \operatorname{Hom}(\mathbf{Q}, \mathbf{L}) \rightarrow \operatorname{Hom}(\mathbf{L}, \mathbf{L}) \rightarrow 0
$$

and from it results the exact cohomology sequence

$$
\begin{aligned}
\cdots & \rightarrow H^{0}(X, \operatorname{Hom}(\mathbf{Q}, \mathbf{L})) \rightarrow H^{0}(X, \operatorname{Hom}(\mathbf{L}, \mathbf{L})) \\
& \stackrel{\delta_{*}}{\rightarrow} H^{1}(X, \operatorname{Hom}(\mathscr{T}, \mathbf{L})) \rightarrow \cdots .
\end{aligned}
$$

There is a canonical element $1 \in H^{0}(X, \operatorname{Hom}(\mathbf{L}, \mathbf{L}))$; the obstruction element $\delta_{*} 1 \in H^{1}(X, \operatorname{Hom}(\mathscr{T}, \mathbf{L}))$ is defined to be the curvature class. On the other hand, there are the isomorphisms $\operatorname{Hom}(\mathscr{T}, \mathbf{L}) \cong \mathscr{T}^{\prime} \otimes \mathbf{L}$ and $H^{1}\left(X,\left(\mathscr{T}^{\prime} \otimes \mathbf{L}\right)\right)$ $\cong H^{0,1}\left(X, \mathscr{T}^{\prime} \otimes \mathbf{L}\right)$. Thus, under the Dolbeault isomorphism, $\delta_{*} 1$ is a global form of type $(1,1)$ with values in $\mathbf{L}(P)$, i.e., a Lie algebra valued form of type $(1,1)$ which is just what a curvature tensor for a complex connexion is. It is of geometrical interest to interpret this curvature class here.

Now let $X: E / F=A / B$ be a $C$-space; associated to the fibering $F \rightarrow E \rightarrow E / F$ we make may the Atiyah construction $0 \rightarrow \mathbf{L} \rightarrow \mathbf{Q} \rightarrow \mathscr{T} \rightarrow 0$; it is easily seen that $\mathbf{L}, \mathbf{Q}, \mathscr{T}$ are the homogeneous vector bundles $E \times_{F} \mathfrak{f}, E \times_{F} \varepsilon, E \times_{F} \varepsilon / \mathfrak{f}$ where $F$ acts on $\mathfrak{f}, \varepsilon$, and $\varepsilon / \mathfrak{f}$ by Ad. Thus $0 \rightarrow \mathbf{L} \rightarrow \mathbf{Q} \rightarrow \mathscr{T} \rightarrow 0$ is obtained from the exact sequence of $F$-modules $0 \rightarrow \mathfrak{f} \rightarrow \boldsymbol{\varepsilon} \rightarrow \boldsymbol{\varepsilon} / \mathfrak{f} \rightarrow 0$ and similarly $0 \rightarrow$ $\operatorname{Hom}(\mathscr{T}, \mathbf{L}) \rightarrow \operatorname{Hom}(\mathbf{Q}, \mathbf{L}) \rightarrow \operatorname{Hom}(\mathbf{L}, \mathbf{L}) \rightarrow 0$ is obtained from the exact sequence of $F$-modules $0 \rightarrow \operatorname{Hom}(\varepsilon / \mathfrak{f}, \mathfrak{f}) \rightarrow \operatorname{Hom}(\varepsilon, \mathfrak{f}) \rightarrow \operatorname{Hom}(\mathfrak{f}, \mathfrak{f}) \rightarrow 0$.

If $V^{\rho} \rightarrow \mathbf{V}^{\rho} \rightarrow E / F$ is a homogeneous vector bundle, $E$ acts on $\mathbf{V}^{\rho}$ and also on $H^{*}\left(X, \mathbf{V}^{\rho}\right)$ where $X=E / F$. In [4] (or also [14]) it is proved that

$$
H^{*}\left(X, \mathbf{V}^{\rho}\right)=\sum_{\lambda} V^{-\lambda} \otimes H^{*}\left(\hat{\mathfrak{n}}, V^{\lambda} \otimes V^{\rho}\right)^{\tilde{\mathfrak{b}}^{\circ}}
$$

where $V^{\lambda}$ runs over all irreducible finite-dimensional $E$-modules, $V^{-\lambda}$ is contragradient to $V^{\lambda}$, and $H^{*}\left(\hat{\mathfrak{n}}, V^{\lambda} \otimes V^{\rho}\right)^{\tilde{\mathbf{b}}^{\circ}}$ is Lie algebra cohomology where $\hat{\mathfrak{n}}=\mathfrak{n} \oplus \overline{\mathfrak{p}} \subset \mathfrak{f}$ acts on $V^{\lambda} \otimes V^{\rho}$ and the notation $H^{*}\left(\hat{\mathfrak{n}}, V^{\lambda} \otimes V^{\rho}\right)^{\tilde{\mathfrak{b}}^{\circ}}$ means that we take $\tilde{\mathfrak{b}}^{\circ}$-invariant cochains.

Theorem 5. Let $X=E / F=A / B$ be a $C$-space. Associated to the fibering $F \rightarrow E \rightarrow E / F$, one constructs the Atiyah sequences

$$
\begin{aligned}
& 0 \rightarrow \mathbf{L} \rightarrow \mathbf{Q} \rightarrow \mathscr{T} \rightarrow 0, \\
& \left.0 \rightarrow \operatorname{Hom}^{\prime} \mathscr{T}, \mathbf{L}\right) \rightarrow \operatorname{Hom}(\mathbf{Q}, \mathbf{L}) \rightarrow \operatorname{Hom}(\mathbf{L}, \mathbf{L}) \rightarrow 0,
\end{aligned}
$$

which are in fact exact sequences of homogeneous vector bundles over $X$. Then, under the requirement of E-invariance, the curvature class $\delta_{*} 1$ in the sense of Atiyah is given (uniquely in the Kähler case) under the Dolbeault isomorphism as the curvature tensor of the canonical complex affine connexion. In particular, the statements of Proposition 6.2 concerning the canonical complex affine connexion are valid for the curvature class in the Atiyah construction.

Proof. The Lie algebra cohomological analogue of the exact sequence 


$$
\begin{aligned}
\cdots & \rightarrow H^{0}(X, \operatorname{Hom}(\mathbf{Q}, \mathbf{L})) \rightarrow H^{0}(X, \operatorname{Hom}(\mathbf{L}, \mathbf{L})) \\
& \stackrel{\delta_{*}}{\rightarrow} H^{1}(X, \operatorname{Hom}(\mathscr{T}, \mathbf{L})) \rightarrow \cdots
\end{aligned}
$$

is

$$
\begin{aligned}
\cdots & \rightarrow V^{-\lambda} \otimes H^{0}\left(\hat{\mathfrak{n}}, V^{\lambda} \otimes \operatorname{Hom}(\varepsilon, \mathfrak{f})\right)^{\tilde{\mathfrak{b}}^{\circ}} \\
& \rightarrow V^{-\lambda} \otimes H^{0}\left(\hat{\mathfrak{n}}, V^{\lambda} \otimes \operatorname{Hom}(\mathfrak{f}, \mathfrak{f}){\tilde{\mathfrak{b}^{\circ}}}^{\tilde{\mathfrak{b}}^{\circ}}\right. \\
& \stackrel{\delta_{*}}{\rightarrow} V^{-\lambda} \otimes H^{1}\left(\hat{\mathfrak{n}}, V^{\lambda} \otimes \operatorname{Hom}(\varepsilon / \mathfrak{f}, \mathfrak{f})\right)^{\tilde{\mathfrak{b}}^{\circ}} \rightarrow \cdots .
\end{aligned}
$$

The requirement that we consider $\varepsilon$-invariant cocycles means that we take $V_{\lambda}$ to be a trivial $\varepsilon$-module. Thus we consider

$$
\begin{aligned}
\cdots & \rightarrow H^{0}(\hat{\mathfrak{n}}, \operatorname{Hom}(\varepsilon, \mathfrak{f}))^{\tilde{\mathbf{b}}^{\circ}} \rightarrow H^{0}(\hat{\mathfrak{n}}, \operatorname{Hom}(\mathfrak{f}, \mathfrak{f}))^{\tilde{\tilde{b}^{\circ}}} \\
& \delta_{*} \\
& \rightarrow H^{1}(\hat{\mathfrak{n}}, \operatorname{Hom}(\varepsilon / \mathfrak{f}, \mathfrak{f}))^{\tilde{\mathfrak{b}}^{\circ}} \rightarrow \cdots
\end{aligned}
$$

(clearly $\left.1 \in H^{0}(\hat{\mathfrak{n}}, \operatorname{Hom}(\mathfrak{f}, \mathfrak{f}))_{\mathfrak{b}^{\circ}}\right)$. To compute $\delta_{*} 1$, we must go to $\operatorname{Hom}(\boldsymbol{\varepsilon}, \mathfrak{f}) \tilde{\mathfrak{b}}^{\circ}$ $=C^{0}(\hat{\mathfrak{n}}, \operatorname{Hom}(\varepsilon, \mathfrak{f}))^{\tilde{\mathfrak{b}}^{\circ}}$ and find a $\pi$ there which extends $1 \in \operatorname{Hom}(\mathfrak{f}, \mathfrak{f})^{\tilde{\mathfrak{b}}^{\circ}}$.

LemMA 7.1. Writing $\varepsilon=\hat{\mathfrak{n}}^{*} \oplus \mathfrak{f}$, a $\pi$ satisfying the above condition is given by the linear projection map of $\varepsilon$ onto $\mathfrak{f}$. In the Kähler case, this $\pi$ is unique.

Proof. It will suffice to prove uniqueness in the Kähler case. If $\pi \in \operatorname{Hom}(\varepsilon, f)^{\tilde{\mathfrak{b}}^{\circ}}$ extends $1 \in \operatorname{Hom}\left(\mathfrak{f}, \mathfrak{f} \tilde{\mathfrak{b}}^{\mathfrak{b}}\right.$, then, since $\mathfrak{h} \subseteq \tilde{\mathfrak{b}}^{\circ}$,

$$
h \circ \pi\left(e_{\alpha}\right)-\pi\left[h, e_{\alpha}\right]=0 \quad \text { for } e_{\alpha} \in \hat{\mathfrak{n}}^{*}\left(=\mathfrak{n}^{*}\right), h \in \mathfrak{h} .
$$

Thus $\left[h, \pi\left(e_{\alpha}\right)\right]=\langle\alpha, h\rangle \pi\left(e_{\alpha}\right)$ for all $h \in \mathfrak{h}$ and thus either $\pi\left(e_{\alpha}\right)=0$ or $\pi\left(e_{\alpha}\right) \in v_{\alpha}$. The rest is clear. Q.E.D.

We observe that $\pi$ is nothing other than the canonical affine connexion interpreted as a cochain. To compute $\delta_{*} 1$, we need only commute $d \pi$. Indeed $d \pi \in \operatorname{Hom}(\hat{\mathfrak{n}}, \operatorname{Hom}(\hat{\mathfrak{n}}, \operatorname{Hom}(\varepsilon / \mathfrak{f}, \mathfrak{f})))$ and for $n, n^{\prime} \in \hat{\mathfrak{n}}, d \pi\left(n^{\prime}\right)(\bar{n})$ lies in $\mathfrak{f}$ and is given by

$$
\begin{aligned}
d \pi\left(n^{\prime}\right)(\bar{n}) & =\left[\bar{n}, \pi\left(n^{\prime}\right)\right]-\pi\left[\bar{n}, n^{\prime}\right] \\
& =-\left[n, \bar{n}^{\prime}\right]_{\mathfrak{f}}=\Xi\left(n^{\prime}, \bar{n}\right)
\end{aligned}
$$

where $\Xi$ is the curvature of the canonical complex affine connexion. Q.E.D.

REMARK. $\pi$ is not unique in the non-Kähler case.

In [8] we shall use Theorem 5 to prove the following theorem.

THEOREM. Let $X$ be a non-Kähler $C$-space and let $T^{2 a} \rightarrow X \rightarrow \hat{X}$ be the toral fibering of $X$ onto a Kähler $C$-space $\hat{X}$. Then there exist a-independent line bundles $\mathbf{L}_{1}, \cdots, \mathbf{L}_{a}$ on $X$ such that $c_{1}\left(\mathbf{L}_{j}\right)=0$ but such that the Atiyah Chern class of $\mathbf{L}_{j}$ is $\neq 0$. 
Remark. This theorem when $X=S \mathscr{U}(3)$ was given by Bott in [4].

VIII. Vanishing theorems on $C$-spaces. The purpose of this section is to use some of the above results to investigate the vanishing mechanism and to try to glean some results in the non-Kähler case. Before doing this, we recall two facts from the theory of complex manifolds [5]. Let $X$ be a compact complex manifold with an Hermitian structure (, ) in $T(X)$. Associated to $($,$) is a (1,1)$-form $\Omega$; $d \Omega=0 \Rightarrow X$ is Kähler. On $A^{p, q}\left(=\right.$ forms of type $(p, q)$ ), one has $L: A^{p, q} \rightarrow A^{p+1, q+1}$ defined by $L(\eta)=\Omega \wedge \eta$, and we define $\Lambda: A^{p, q} \rightarrow A^{p-1, q-1}$ by $(L \eta, \phi)=(\eta, \Lambda \phi)$. Then

$$
\Lambda L-L \Lambda=(n-p-q) I \quad \text { on } A^{p, q},
$$

where $I$ is the identity. If $V \rightarrow \mathbf{V} \rightarrow X$ is an analytic vector bundle over a Kähler manifold $X$, then if $\mathbf{V}$ has a Hermitian metric $h$, one has as usual a pairing (,) between $A^{p, q}(\mathbf{V})$ and $A^{n-q, n-p}\left(\mathbf{V}^{\prime}\right)$. As mentioned above, $\theta=h^{-1} \partial h$ gives a connexion in $\mathbf{V}$ and covariant differentiation on local sections of $\mathbf{V}$ is given by

$$
D=D^{\prime}+\bar{\partial}
$$

where $D^{\prime}=\partial+e(\theta)$. If we define $\vartheta$ and $\vartheta^{\prime}$ by $(\phi, \vartheta \eta)=(\bar{\partial} \phi, \eta),\left(\phi, \vartheta^{\prime} \eta\right)=\left(D^{\prime} \phi, \eta\right)$, then $\square=\bar{\partial} \vartheta+\vartheta \bar{\partial}$ gives a Laplacian in $A^{p, q}(\mathbf{V})$ and

$$
\Lambda \bar{\partial}-\bar{\partial} \Lambda=-\sqrt{ }-1 \vartheta^{\prime}
$$

which is the fundamental Kähler identity. Finally, the Ricci identity reads:

$$
D^{2}=\left(D^{\prime}+\bar{\partial}\right)^{2}=D^{\prime} \bar{\partial}+\bar{\partial} D^{\prime}=e(\Xi)
$$

where $\Xi=\bar{\partial} \theta$ is the curvature form (see [7]).

Proposition 8.1 (NAKano). $\sqrt{ }-1(\Lambda e(\Xi) \phi, \phi) \geqq 0$ for $\phi$ harmonic, $\phi \in A^{p, q}(\mathbf{V})$.

\section{Proof.}

$$
\begin{aligned}
\sqrt{ }-1(\Lambda e(\Xi) \phi, \phi) & =\sqrt{ }-1\left(\Lambda \bar{\partial} D^{\prime} \phi, \phi\right) \\
& =\sqrt{ }-1\left(\Lambda \bar{\partial} D^{\prime} \phi, \phi\right)-\sqrt{ }-1\left(D^{\prime} \phi, L \vartheta \phi\right) \\
& =\sqrt{ }-1((\Lambda \bar{\partial}-\bar{\partial} \Lambda) \phi, \phi)=\sqrt{ }-1\left(-\sqrt{ }-1\left(\vartheta^{\prime} D^{\prime}\right) \phi, \phi\right) \\
& =\left(D^{\prime} \phi, D^{\prime} \phi\right) \geqq 0 . \quad \text { Q.E.D. }
\end{aligned}
$$

Dually, one proves that $\sqrt{ }-1(e(\Xi) \Lambda \phi, \phi) \leqq 0$ and by subtraction, $\sqrt{ }-1((\Lambda e(\Xi)-e(\Xi) \Lambda) \phi, \phi) \geqq 0$.

Now let $V^{\rho} \rightarrow \mathbf{V}^{\rho} \rightarrow X=A / B=E / F$ be a homogeneous line bundle over a Kähler $C$-space $X$. Then $\mathfrak{f}=\mathfrak{b}_{\Psi} \oplus \mathfrak{h}_{\Psi} \oplus \mathfrak{n}$ and $\rho \mid \mathfrak{b}_{\Psi} \oplus \mathfrak{n}=0$, i.e., $\rho$ is a complexification of a character on the center of $B$. We choose a positive-definite Kähler metric $Q_{\eta}$ by (5.2) on $X$ so that $Q_{\eta}\left(e_{\alpha}, e_{\beta}\right)=\delta_{\beta}^{\alpha} Q(\eta, \alpha)$ for $\alpha, \beta \in \Sigma^{+}-\Psi^{+}$. We denote 
the Cartan-Killing form $Q$ by (, ) and for $\alpha \in \Sigma^{+}-\Psi^{+}$we set $f_{\alpha}=e_{\alpha} / \sqrt{ }(\eta, \alpha)$ so that

$$
Q_{\eta}\left(f_{\alpha}, f_{\beta}\right)=\delta_{\beta}^{\alpha}, \Xi_{\rho}\left(f_{\alpha}, f_{-\beta}\right)=-\delta_{\beta}^{\alpha} \rho\left(\left(h_{\alpha}\right)_{\mathfrak{h}_{\Psi}}\right) /(\eta, \alpha) \text { by }(6.2)
$$

where $h_{\alpha}=\left[e_{\alpha}, e_{-\alpha}\right]$ and ()$_{\mathfrak{h}_{\Psi}}$ signifies the projection of $\mathfrak{h}$ on $\mathfrak{h}_{\Psi}$. If $\omega^{\alpha}$ is dual to $f_{\alpha}$,

and

$$
d s^{2}=\sum_{\alpha \in \Sigma^{+}-\Psi^{+}} \omega^{\alpha} \circ \bar{\omega}^{\alpha}
$$

$$
\Omega=\frac{\sqrt{ }-1}{2} \sum_{\alpha \in \Sigma^{+}-\Psi^{+}} \omega^{\alpha} \wedge \bar{\omega}^{\alpha} .
$$

Finally, we remark that $\Xi_{\rho}$ given above is the curvature of a metric connexion by Proposition 6.4 and thus (8.3) and Proposition 8.1 both hold for $\Xi_{\rho}$.

Proposition 8.2. If $\rho\left(h_{\alpha}\right)<0$ for all $\alpha \in \Sigma^{+}-\Psi^{+}$, then there are no harmonic forms in $A^{0, q}(\mathbf{V})$ for $0 \leqq q<n$.

Proof. Since the metric and curvature are $A$-invariant, we may calculate at the origin ( = coset of the identity). There $\phi=(1 / q !) \phi_{\beta_{1} \ldots \beta_{q}} \bar{\omega}^{\beta_{1}} \wedge \cdots \wedge \bar{\omega}^{\beta_{q}}$ where $\phi_{\beta_{1} \ldots \beta_{q}}$ transforms in $\mathbf{V}^{\rho} \otimes\left(\wedge^{q} \mathscr{T}^{\prime}(X)\right)$. By a routine calculation which need not be written out,

$$
\Lambda e\left(\Xi_{\rho}\right) \phi=-\frac{\sqrt{ }-1}{2(q !)}\left(\sum_{\alpha \neq \beta_{1} \ldots \beta_{n}} \frac{\rho\left(\left(h_{\alpha}\right)_{\mathfrak{h}_{\Psi}}\right)}{(\eta, \alpha)} \phi_{\beta_{1} \ldots \beta_{n}} \bar{\omega}^{\beta_{1}} \wedge \cdots \wedge \bar{\omega}^{\beta_{q}}\right)
$$

and the Nakano inequality implies that

$$
\sqrt{ }-1 \int_{X}\left(\Lambda e\left(\Xi_{\rho}\right) \phi\right) h \wedge * \bar{\phi} \geqq 0 .
$$

Up to a positive constant, the integrand of this expression evaluated at the origin is

$$
\sum_{\alpha \neq \beta_{1} \ldots \beta} \frac{\rho\left(\left(h_{\alpha}\right)_{\mathfrak{b} \Psi}\right)}{(\eta, \alpha)}\left|\phi_{\beta_{1} \ldots \beta_{a}}\right|^{2}
$$

The proposition follows from this.

COROLLARY. If $\rho\left(\left(h_{\alpha}\right)_{\mathfrak{h}_{\Psi}}\right) \leqq 0$ for $\alpha \in \Sigma^{+}-\Psi^{+}$and if for one such $\alpha, \rho\left(\left(h_{\alpha}\right)_{\mathfrak{h}_{\psi}}\right)<0$, then there are no harmonic forms in $A^{0,0}\left(V^{\rho}\right)$.

We may now ask about harmonic forms in $A^{p, q}\left(\mathbf{V}^{\rho}\right)$.

PROPOSITION 8.3. If $\rho\left(h_{\alpha}\right)<0$ for $\alpha \in \Sigma^{+}-\Psi^{+}$, then there are no harmonic forms $\phi \in A^{p, q}\left(\mathbf{V}^{\rho}\right)$ for $p+q \leqq n-1$.

Proof. This may be done exactly the same way as Proposition 8.2; we proceed somewhat differently. If $\rho$ satisfies the given hypothesis, then we define $Q_{\rho}$ by $Q_{\rho}\left(e_{\alpha}, e_{-\beta}\right)=-\delta_{\beta}^{\alpha} \rho\left(h_{\alpha}\right)$ (note that $\rho\left(h_{\alpha}\right)=0$ for $\alpha \in \Psi^{+}$). Then 
and

$$
d s^{2}=\sum_{\alpha \in \Sigma^{+}-\Psi^{+}}-\rho\left(h_{\alpha}\right) \omega^{\alpha} \circ \bar{\omega}^{\alpha}
$$

and thus

$$
\Omega=\frac{\sqrt{ }-1}{2} \sum_{\alpha \in \Sigma^{+}-\Psi^{+}}-\rho\left(h_{\alpha}\right) \omega^{\alpha} \wedge \bar{\omega}^{\alpha}
$$

$$
\Xi_{\rho}=-\frac{\lambda}{\sqrt{ }-1} \Omega \quad \text { where } \lambda>0
$$

By (8.1) and the remark after Proposition 8.1,

$$
\begin{aligned}
0 & \leqq \sqrt{ }-1\left(\left(\Lambda e\left(\Xi_{\rho}\right)-e\left(\Xi_{\rho}\right) \Lambda\right) \phi, \phi\right)=-\lambda((\Lambda L-L \Lambda) \phi, \phi) \\
& =-\lambda(n-p-q)(\phi, \phi) . \quad \text { Q.E.D. }
\end{aligned}
$$

We have proven the following theorem, most of which is due to Borel and Bott.

THEOREM 6. Let $V^{\rho} \rightarrow V^{\rho} \rightarrow X$ be a homogeneous line bundle over a Kähler $C$-space $X=A / B=E / F$. Then if $\rho$ is nonpositive and has at least one negative eigenvalue on the center of $\mathrm{b}^{\circ}, H^{0}\left(X, \mathrm{~V}^{\rho}\right)=0$. If $\rho$ is strictly negative on the center of $\mathfrak{b}^{\circ}, H^{p, q}\left(X, V^{\rho}\right)=H^{q}\left(X, \Omega^{p} \otimes V^{\rho}\right)=0$ for $p+q \leqq n-1$ where $\Omega^{p}=\left(\Lambda^{p} \mathscr{T}^{\prime}(X)\right)$.

In the non-Kähler case, one has (8.1) but (8.2) is no longer true. Whereas in the Kähler situation, the Nakano inequality is an expression of the form $(R \phi, \phi) \geqq 0$ ( $\phi$ harmonic) where $R$ is an operator involving only second order or curvature terms, in the non-Kähler case one arrives at an expression of the form

$$
(R \phi, \phi)+(\chi(\phi), \phi) \geqq 0 \quad(\phi \text { harmonic })
$$

where $\chi$ is an operator in the first order or connexion terms.

THEOREM 6'. Let $E^{\rho} \rightarrow \mathbf{E}^{\rho} \rightarrow X$ be a homogeneous line bundle over a $C$-space $X$ where $\operatorname{dim}_{\mathbf{C}} X=n$ ( $X$ may be Kähler or not). Suppose that the first Chern class $c_{1}\left(\mathbf{E}^{\rho}\right)$ is given by a negative semi-definite quadratic form of index $k \leqq n$. Then

$$
H^{q}\left(X, \mathscr{E}^{\rho}\right)=0 \quad(q<k) .
$$

Remarks. If $k=n, X$ is necessarily Kähler and we recover Proposition 8.2. The idea of the proof of (8.5) is contained in the proof of Proposition 8.2; the details are somewhat more complicated. We remark that using (8.5) it is not too hard to give a purely differential-geometric proof for line bundles of the main theorem of Bott (Theorem IV' in [4]). Our main interest is in the non-Kähler case and we shall give this result here, deferring its proof together with the proof of (8.5) to a later paper.

Let $X=G / \mathscr{U}$ be a non-Kähler $C$-space and suppose that $T^{2 a} \rightarrow X \stackrel{\pi}{\rightarrow} X$ is the toral fibering of $X$ over a Kähler $C$-space $\hat{X}=G / \hat{\mathscr{U}}$. Then $\mathscr{U}$ is a normal 
subgroup of $\hat{\mathscr{U}}$, we have $\rho: \hat{\mathscr{U}} \rightarrow \hat{\mathscr{U}} / \mathscr{U} \cong T^{2 a}$, and the manifold $X$ is given as the homogeneous torus bundle $G \times \hat{\mathscr{u}} T^{2 a}$. This bundle is an analytic principal bundle associated to the principal bundle $\hat{\mathscr{U}} \rightarrow G \rightarrow G / \hat{\mathscr{U}}$; thus $T^{2 a} \rightarrow X \rightarrow \hat{X}$ inherits the canonical complex connexion (Definition 6.2) in $\hat{\mathscr{U}} \rightarrow G \rightarrow G / \hat{\mathscr{U}}$. Letting $\mathfrak{p}=$ complex Lie algebra of $T^{2 a}$, the connexion form $\omega$ is a $\mathfrak{p}$-valued $(1,0)$ form on $X$ and, choosing an isomorphism $\mathfrak{p} \cong \mathbf{C}^{a}$, we may write $\omega=\omega_{1}+\cdots+\omega_{a}$ where the $\omega_{j}$ are scalar-valued $(1,0)$ forms on $X$. The curvature form $\Xi$ may be written $\Xi=\Xi_{1}+\cdots+\Xi_{a}$ where $\Xi_{j}=d\left(\omega_{j}\right)=\bar{\partial}\left(\omega_{j}\right)$ (since $\Xi_{j}$ is of type $(1,1)$ ). This implies that $\partial \omega_{j}=0$ and because the connexion is not integrable, $\Xi_{j} \neq 0$. The forms $\bar{\omega}_{j}$ are thus invariant (since the connexion is) $(0,1)$ forms which are $\bar{\partial}$-closed but are not $d$-closed.

Let $E^{\hat{\rho}} \rightarrow \mathbf{E}^{\hat{\rho}} \rightarrow \hat{X}$ be a homogeneous line bundle; in [4] Bott showed that $H^{q}\left(\hat{X}, \mathscr{E}^{\hat{\rho}}\right) \neq 0$ for at most one $q=q_{0}$ and $H^{q_{0}}\left(\hat{X}, \mathscr{E}^{\hat{\rho}}\right)$ is an irreducible $G$-module with induced representation denoted by $\hat{\rho}^{*}$. The restriction of $\hat{\rho}$ to $\mathscr{U}$ gives $\rho: \mathscr{U} \rightarrow \mathrm{GL}\left(E^{\rho}\right)$ and we may form $E^{\rho} \rightarrow \mathbf{E}^{\rho} \rightarrow G / \mathscr{U}$; it is not hard to show that $\pi^{-1}\left(\mathbf{E}^{\hat{\rho}}\right)=\mathbf{E}^{\rho}$ where $\pi^{-1}=$ lifting of bundles. Thus if $\xi$ is an $\mathbf{E}^{\hat{\rho}}$-valued form representing a cohomology class in $H^{q_{0}}\left(\hat{X}, \mathscr{E}^{\hat{\rho}}\right) \cong H^{0, q_{0}}\left(\hat{X}, \mathbf{E}^{\hat{\rho}}\right), \pi^{*}(\xi)$ is defined and represents a class in $H^{0, q}\left(X, \mathbf{E}^{\rho}\right)$.

THEOREM 7. $H^{q}\left(X, \mathscr{E}^{\rho}\right)=0$ if $q<q_{0}$ a nd $H^{q_{0}+p}\left(X, \mathscr{E}^{\rho}\right) \cong H^{q_{0}}\left(\hat{X}, \mathscr{E}^{\hat{\rho}}\right) \otimes H^{p}(X, \Omega)$. The induced representation is $(\hat{\rho})^{*} \otimes 1$, and the forms representing $H^{q_{0}+p}\left(X, \mathscr{E}^{\rho}\right) \cong H^{0, q_{0}+p}\left(X, \mathbf{E}^{\rho}\right)$ may be chosen to be $\pi^{*}(\xi) \otimes \bar{\omega}_{i_{1} \ldots i_{p}}$ where $\bar{\omega}_{i_{1} \ldots i_{p}}=\bar{\omega}_{i_{1}} \wedge \cdots \wedge \bar{\omega}_{i_{p}}$ and the $\bar{\omega}_{j}$ and $\xi$ were defined above.

This shows how the canonical connexion enters into the discussion of the groups $H^{q}\left(X, \mathscr{E}^{\rho}\right)$. The proofs of Theorems $6^{\prime}$ and 7 will be given in [8] and the interpretation of the forms will rely upon the results of this paper.

IX. Some applications to sheaf theory. To the best of the author's knowledge, the main applications of differential geometry to complex manifold theory have been to the study of characteristic classes and to vanishing theorems. We shall now indicate some other (less profound) applications which may be of interest.

(i) Let $X=G / U$ (complex form) $=M / V$ (compact form) be a $C$-space, and let $p: U \rightarrow U^{\prime} \subset \mathrm{GL}\left(E^{\rho}\right)$ be a holomorphic representation. Then we may construct the homogeneous principal bundle

$$
U^{\prime} \rightarrow P^{\prime}=P \times_{U} U^{\prime} \rightarrow X
$$

and the associated vector bundle

$$
E^{\rho} \rightarrow \mathbf{E}^{\rho}=P^{\prime} \times_{U^{\prime}} E^{\rho} \rightarrow X .
$$

Forming the Atiyah sequence ( $\$ 6)$ associated to (9.1), we get

$$
0 \rightarrow \mathbf{L}\left(P^{\prime}\right) \rightarrow \mathbf{Q}\left(P^{\prime}\right) \rightarrow T(X) \rightarrow 0 .
$$


From Theorem 5, we have

Proposition 9.1. The obstruction to splitting (9.3) is given by $\Xi^{\prime}=p_{*}(\Xi) \in H^{1}\left(X, \operatorname{Hom}\left(T(X), \mathbf{L}\left(P^{\prime}\right)\right)\right)$ where $\Xi$ is the curvature tensor of the canonical complex connexion.

The bundle $T\left(P^{\prime}\right) / U^{\prime}=\mathbf{Q}\left(P^{\prime}\right)$ is a homogeneous vector bundle given by some linear action of $U$ : we shall determine this representation. Note that $\mathbf{L}\left(P^{\prime}\right)$ is given by the linear representation $\operatorname{Ad} \circ \rho$ of $U$ on $\mathfrak{u}^{\prime}$. A typical fibre of $\mathbf{Q}\left(P^{\prime}\right)$ is $E=\mathfrak{u}^{\prime} \oplus \mathfrak{g} / \mathfrak{u}$, and because of (9.3), the representation of $\mathfrak{u}$ on $E$ must be of the form

$$
u \rightarrow\left(\begin{array}{cc}
\operatorname{ad}(u) & \zeta(u) \\
0 & \operatorname{ad} u
\end{array}\right) \quad \text { for some } \zeta \operatorname{Hom}\left(\mathfrak{u}, \operatorname{Hom}\left(\mathfrak{g} / \mathfrak{u}, \mathfrak{u}^{\prime}\right)\right)
$$

Proposition 9.2. Write $\mathfrak{u}=\tilde{\mathfrak{v}}^{\circ} \oplus \mathfrak{n}$. Then the element $\zeta \in \operatorname{Hom}\left(\mathfrak{u}, \mathrm{m}\left(\mathfrak{g} / \mathfrak{u}, \mathfrak{u}^{\prime}\right)\right)$ in (9.4) is given as follows:

$$
\zeta(v)=0 \text { for } v \in \tilde{\mathfrak{v}}^{\circ} \text { and } \zeta(n) g^{\prime}=\Xi^{\prime}(g, n) \text { for } n \in \mathfrak{H}, g \in \mathfrak{g} / \mathfrak{u} \text {. }
$$

Thus we may write the representation of $u$ in the form

$$
\left(\begin{array}{cc}
\text { ad } & \Xi^{\prime} \\
0 & \text { ad }
\end{array}\right)
$$

Proof. If we consider the case of the principal bundle $U \rightarrow G \rightarrow G / U$, then $E=\mathfrak{u} \oplus \mathfrak{g} / \mathfrak{u} \cong \mathfrak{g}$ (as vector spaces) and the representation (9.5) is just the adjoint representation of $\mathfrak{u}$ on $\mathfrak{g}$, since $\Xi$ is the curvature of the canonical complex connexion. The general case follows from this, coupled with mapping properties of the Atiyah construction. Q.E.D.

Now the homogeneous bundle $\mathbf{E}$ has a holomorphic connexion if and only if (9.3) splits analytically, which can be done if and only if in the cohomology sequence

$$
\cdots \rightarrow H^{0}\left(X, \operatorname{Hom}\left(T(X), Q\left(P^{\prime}\right)\right)\right) \stackrel{\pi}{\rightarrow} H^{0}(X, \operatorname{Hom}(T(X), T(X))) \rightarrow \cdots
$$

there exists a $\chi \in H^{0}\left(X, \boldsymbol{H o m}\left(T(X), \mathbf{Q}\left(P^{\prime}\right)\right)\right)$ such that $\pi(\chi)=1$. Now (9.6) is an exact sequence of $M$-modules, and if such a $\chi$ exists, it may be chosen to be $M$-invariant. It then follows, by (7.2), that (9.3) splits analytically if and only if the exact sequence of $\mathfrak{u}$-modules $0 \rightarrow \mathfrak{u}^{\prime} \rightarrow E \rightarrow \mathfrak{g} / \mathfrak{u} \rightarrow 0$ splits. Thus, by Proposition 9.2 , we conclude that, by redefining the action of $\mathfrak{u}$ by a change of coordinates if necessary, (9.3) splits analytically if and only if the canonical complex connexion is integrable. Since $\pi_{1}(X)=1$, we have

THEOREM 8. A homogeneous vector bundle has a holomorphic connexion if and only if it is analytically trivial. 
(ii) The results in this section are valid for arbitrary complex manifolds. Let $X$ be a complex manifold and let

$$
(S): 0 \rightarrow \mathbf{E}^{\prime} \rightarrow \mathbf{E} \rightarrow \mathbf{E}^{\prime \prime} \rightarrow 0
$$

be an exact sequence of analytic vector bundles over $X$. Then there is associated to $(S)$ an element, unique to nonzero multiples, $\zeta_{(s)} \in H^{1}\left(X, \operatorname{Hom}\left(\mathbf{E}^{\prime \prime}, \mathbf{E}^{\prime}\right)\right)$ such that $(S)$ splits analytically if and only if $\zeta_{(s)}=0$. If $U=\left\{U_{i}\right\}$ is a Stein covering of $X$ with nerve $N$, and if the transition functions of $E^{\prime}, E^{\prime \prime}$ are $\left\{e_{i j}^{\prime}\right\},\left\{e_{i j}^{\prime \prime}\right\}$, then the transition functions of $E$ may be written as

$$
e_{i j}=\left(\begin{array}{rr}
e_{i j}^{\prime} & \sigma_{i j} \\
0 & e_{i j}^{\prime \prime}
\end{array}\right)
$$

and $\zeta_{(s)} \in H^{1}\left(N, \operatorname{Hom}\left(\mathbf{E}^{\prime \prime}, \mathbf{E}^{\prime}\right)\right)$ is given by $\zeta_{i j}=\sigma_{i j}\left(e_{i j}^{\prime \prime}\right)^{-1}$.

Now if $A, B, C$ are analytic sheaves over $X$, and if there is a pairing $A \otimes_{\Omega} B \rightarrow C$, then there is defined a pairing

$$
\circ: C^{p}(N, A) \otimes C^{q}(N, B) \rightarrow C^{p+q}(N, C)
$$

such that

$$
\delta(\zeta \circ \eta)=\delta(\zeta) \circ \eta+(-1)^{p} \zeta \circ \delta(\eta)
$$

where $\zeta \in C^{p}(N, A), \eta \in C^{q}(N, B)$. The definition of this pairing is the same formally as for constant sheaves. By (9.10) there is a cup-product

$$
\circ: H^{p}(N, A) \otimes H^{q}(N, B) \rightarrow H^{p+q}(N, C) \text {. }
$$

In particular, there is a cup-product

$$
\circ: H^{1}\left(X, \boldsymbol{H o m}\left(\mathbf{E}^{\prime \prime}, \mathbf{E}^{\prime}\right)\right) \otimes H^{q-1}\left(X, \mathscr{E}^{\prime \prime}\right) \rightarrow H^{q}\left(Y, \mathscr{E}^{\prime}\right) .
$$

Proposition 9.3. In the exact cohomology sequence of (9.7), the coboundary mappings $\delta^{q-1}: H^{q-1}\left(Y, \mathscr{E}^{\prime \prime}\right) \rightarrow H^{q}\left(Y, \mathscr{E}^{\prime}\right)$ are given by

$$
\delta^{q-1}(\eta)=\zeta_{(s)} \circ \eta \text { for } \eta \in H^{q-1}\left(Y, \mathscr{E}^{\prime \prime}\right) .
$$

Furthermore, ${ }^{\circ}$ may be interpreted either in terms of $\check{C} e c h$ or Dolbeault cohomology.

Proof. Write $\mathscr{F}^{\prime}=\mathscr{E}^{\prime}, \mathscr{F}=\mathscr{E}, \mathscr{F}^{\prime \prime}=\mathscr{E}^{\prime \prime}, C^{q}\left(\mathscr{F}^{\prime}\right)=C^{q}\left(N, \mathscr{F}^{\prime}\right)$, etc., and consider the diagram

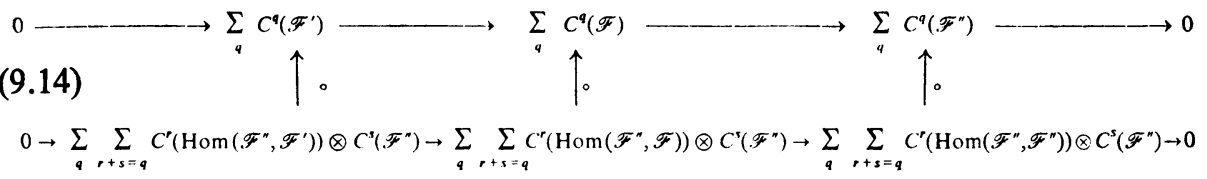


By defining $\delta$ on $C^{r}\left(\operatorname{Hom}\left(\mathscr{F}^{\prime \prime}, \mathscr{E}\right)\right) \otimes C^{s}\left(\mathscr{F}^{\prime \prime}\right)\left(\mathscr{E}=\mathscr{F}^{\prime}, \mathscr{F}, \mathscr{F}^{\prime \prime}\right)$ to be $\delta=\delta \otimes 1+$ $(-1)^{r} 1 \otimes \delta$, then by $(9.10),(9.14)$ is a commutative diagram of chain complexes. Thus we have



Now the mapping

$$
\begin{aligned}
\delta^{q-1}: H^{r}\left(\operatorname{Hom}\left(\mathscr{F}^{\prime \prime}, \mathscr{F}^{\prime \prime}\right)\right) \otimes H^{s}\left(\mathscr{F}^{\prime \prime}\right) \rightarrow & H^{r+1}\left(\operatorname{Hom}\left(\mathscr{F}^{\prime \prime}, \mathscr{F}^{\prime}\right)\right) \otimes H^{s}\left(\mathscr{F}^{\prime \prime}\right) \\
& \oplus H^{r}\left(\operatorname{Hom}\left(\mathscr{F}^{\prime \prime}, \mathscr{F}^{\prime \prime}\right)\right) \otimes H^{q+1}\left(\mathscr{F}^{\prime \prime}\right)
\end{aligned}
$$

is simply $\delta^{r} \otimes 10+0$ where $\delta^{r}: H^{r}\left(\operatorname{Hom}\left(\mathscr{F}^{\prime \prime}, \mathscr{F}^{\prime \prime}\right)\right) \rightarrow H^{r+1}\left(\operatorname{Hom}\left(\mathscr{F}^{\prime \prime}, \mathscr{F}^{\prime}\right)\right)$ is the usual coboundary. Thus, if $\eta \in H^{q-1}\left(\mathscr{F}^{\prime \prime}\right), H^{0}\left(\operatorname{Hom}\left(\mathscr{F}^{\prime \prime}, \mathscr{F}^{\prime \prime}\right)\right)$, then $\delta^{q-1}\left(\zeta^{\circ} \eta\right)=\delta^{0}(\zeta)^{\circ} \eta$, and taking $\zeta=1 \in H^{0}\left(\operatorname{Hom}\left(\mathscr{F}^{\prime \prime}, \mathscr{F}^{\prime \prime}\right)\right)$, we get the first part of the proposition. The second part follows, e.g., from the general remarks in the last chapter of the book by Godement on sheaves. Q.E.D.

Now let $Y$ be compact and let $A \rightarrow P \rightarrow Y$ be an analytic principal bundle where $A$ is a complex Lie group. Let $\theta \in H^{0}\left(Y,(T(Y))^{\sim}\right)$ be a global holomorphic vector field on $Y$, and set $\theta(t)=\exp (t \theta)$. Then we clearly have:

Proposition 9.4. Let $0 \rightarrow \mathbf{L}(P) \rightarrow \mathbf{Q}(P) \rightarrow^{\pi} T(Y) \rightarrow 0$ be the Atiyah sequence of $A \rightarrow P \rightarrow Y$. Then $\theta(t)$ lifts to bundle action in $P$ if and only if there exists a section $\psi \in H\left(Y,(\mathbf{Q}(P))^{\sim}\right)$ such that $\pi(\psi)=\theta \in H\left(Y, T(Y)^{\sim}\right)$.

Proposition 9.5. In order that $\theta(t)$ lift in action in $P$, it is both necessary and sufficient that, for any connexion $\omega$ of type $(1,0)$ in $P$ with $\bar{\partial} \omega=\Xi$, we have

$$
i(\theta) \Xi=\bar{\partial} \Phi_{\theta} \text { for some } C^{\infty} \text { section } \Phi_{\theta} \text { of } \mathbf{L}(P) \text {. }
$$

(Here $i$ is the operation of contraction.)

Proof. By Proposition 9.3, $\delta^{\circ}(\theta)=i(\theta) \Xi$, and we may use Proposition 9.4. Q.E.D.

REMARK. Equation (9.15) will have applications to variation of complex structure. 


\section{BIBLIOGRA PHY}

1. M. F. Atiyah, Complex connexions in fibre bundles, Trans. Amer. Math. Soc. 85, (1957), 181-207.

2. W. Boothby, Some fundamental formulas for Hermitian manifolds with non-vanishing torsion, Amer. J. Math. 76 (1954), 509-534.

3. A. Borel and F. Hirzebruch, Characteristic classes of homogeneous spaces. I, Amer. J. Math. 80 (1958), 459-538.

4. R. Bott, Homogeneous vector bundles, Ann. of Math. (2) 66 (1957), 203-248.

5. E. Calabi and E. Vesentini, On compact, locally symmetric Kähler manifolds, Ann. of Math. (2) 71 (1960), 472-507.

6. S. S. Chern, Characteristic classes of Hermitian manifolds, Ann. of Math. (2) 47 (1946), 85-121.

7. P. A. Griffiths, On a theorem of Chern, Illinois J. Math. 6 (1962), 468-479.

8. - On certain homogeneous complex manifolds, (to appear).

9. J. Hano and S. Kobayashi, A fibering of a class of homogeneous complex manifolds, Trans. Amer. Math. Soc. 94 (1960), 233-243.

10. J. Hano and Y. Matsushima, Some studies on Kaehlerian homogeneous spaces, Nagoya Math. J. 11 (1957), 77-92.

11. S. Kobayashi, On compact Kähler manifolds with positive definite Ricci tensor, Ann. of Math. (2) 74 (1961), 570-574.

12. K. Kodaira, On a differential-geometric method in the theory of analytic stacks, Proc. Nat. Acad. Sci. U. S. A. 39 (1953), 868-872.

13. J. L. Koszul, Sur la forme hermitienne canonique des espaces homogènes complexes, Canad. J. Math. 7 (1955), 562-576.

14. B. Kostant, Lie algebra cohomology and the generalized Borel-Weil theorem, Ann. of Math. (2) 74 (1961), 329-387.

15. K. Nomizu, Invariant affine connexions on homogeneous spaces, Amer. J. Math. 76 (1954), 33-65.

16. Lie groups and differential geometry, The Mathematical Society of Japan, Japan, 1956.

17. I Singer, The geometric interpretation of a special connection, Pacific J. Math. 9 (1959), 585-590.

18. H. C. Wang, Closed manifolds with homogeneous complex structure, Amer. J. Math. 76 (1954), 1-32.

19. - - On invariant connections over a principal fibre bundle, Nagoya Math. J. 13 (1958), 1-19.

PRINCETON UNIVERSITY,

Princeton, New Jersey 